\title{
Global robust output regulation for a class of affine singular nonlinear systems
}

\author{
Bomin Huang, Lingmei Chen, and Weiyao Lan* \\ Department of Automation, Xiamen University, Xiamen 361005, China
}

\begin{abstract}
The global robust output regulation problem of the singular nonlinear system is investigated. Motivated by the inputoutput linearization of the normal affine nonlinear system, a global diffeomorphism map is designed under the assumption that the singular nonlinear system has a strong relative degree. The global diffeomorphism map transfers the singular nonlinear system into a new singular nonlinear system with a special structure. Attaching an internal model to the new singular nonlinear system yields an augmented singular nonlinear system and the global robust stabilization solution of the augmented system implies the global robust output regulation solution of the original singular nonlinear system. Then the global stabilization problem is solved by some appropriate assumptions and the solvability conditions of the global robust output regulation problem are established. Finally, a simulation example is given to illustrate the design approach.
\end{abstract}

Keywords: singular nonlinear system, diffeomorphism map, output regulation, robust control.

DOI: $10.21629 /$ JSEE.2017.04.14

\section{Introduction}

A large number of physical systems can be modeled by singular nonlinear systems, e.g., power systems [1], robot systems [2] and chemical processes [3]. Hence, the control problems for singular nonlinear systems attract much research attention. For example, [4] and [5] addressed the issues of input-output decoupling and disturbances decoupling for singular nonlinear systems; [6] dealed with the adaptive regularization problem of singular nonlinear systems, and the tracking problem was investigated in [7].

The robust nonlinear output regulation problem aims to achieve asymptotic tracking of a class of reference inputs and/or asymptotic rejecting of a class of disturbances for nonlinear systems in the presence of parameter uncertainties. Most of the results on the robust nonlinear output

Manuscript received April 12, 2016.

*Corresponding author.

This work was supported by the National Natural Science Foundation of China (61374035) and the Fundamental Research Funds for the Central Universities (20720150177). regulation problem are based on two methods. One is internal model principle, for example, linear internal model [8 12], and nonlinear internal model [13]. The other one is the adaptive method, for example, adaptive internal model [14,15], and adaptive output regulation [16-19]. Besides the output regulation problem for normal nonlinear systems, the output regulation problem for singular nonlinear systems has also been investigated in the literature, for example, [20-25]. It is shown in [20] that a necessary condition for the output regulation problem of singular nonlinear systems is that the singular nonlinear regulator equations have a nontrivial solution. Under an assumption on normalizability, a state feedback controller and an output feedback controller were designed in [20] to solve the output regulation problem. Moreover, [22] and [23] removed the normalizability condition in [20], and designed a normal output feedback control law for the output regulation problem of the singular nonlinear systems. The robust output regulation problem for singular nonlinear systems was studied in [21] and [24]. However, it should be noted that the existing results on the robust output regulation problem for singular nonlinear systems are limited to local solutions because the solvability conditions on the robust output regulation problem for singular nonlinear systems are generally obtained by the linearization model of the singular nonlinear systems.

In this paper, we are trying to investigate the global robust output regulation problem for a class of affine singular nonlinear systems. Noting that it is almost impossible to solve the global robust output regulation problem directly for a general singular nonlinear system, a global diffeomorphism map is designed to transfer the singular nonlinear system into a new singular nonlinear system with a special structure. Such a global diffeomorphsim map exists under the assumption that the singular nonlinear system has a strong relative degree. The global robust output regulation problem is transferred into a global robust stabilization problem. And then, the solvability conditions 
of the global robust output regulation problem are established by solving the global robust stabilization problem with the virtue of the special structure. Specifically, we attach a dynamic compensator to the new singular nonlinear system which yields an augmented singular nonlinear system. It is shown that the solvability of the robust stabilization for the augmented singular nonlinear system implies the solvability of the robust output regulation problem of the original singular nonlinear system. Under some appropriate assumptions, the global robust stabilization problem is solved with a state feedback control law by using the small gain theorem. Then, the solvability conditions of the global robust output regulation problem are established.

The rest of this paper is organized as follows. Section 2 formulates the global robust output regulation problem for a class of singular nonlinear systems. Section 3 transforms the singular nonlinear system in the affine form into a singular nonlinear system with special structure, and the output regulation problem is converted into a robust stabilization problem of an augmented singular nonlinear system. Section 4 addresses the solvability conditions for the global robust stabilization problem of the augmented singular nonlinear system. Section 5 gives an example to illustrate the proposed method, and some remark conclusions are contained in Section 6.

\section{Problem formulation}

Consider the following affine singular nonlinear system:

$$
\begin{gathered}
\dot{\boldsymbol{x}}=\boldsymbol{f}_{1}(\boldsymbol{x}, \boldsymbol{\mu})+\boldsymbol{p}_{1}(\boldsymbol{x}, \boldsymbol{\mu}) \boldsymbol{z}+\boldsymbol{g}_{1}(\boldsymbol{x}, \boldsymbol{\mu}) \boldsymbol{u}+\boldsymbol{q}_{1}(\boldsymbol{x}, \boldsymbol{\mu}) \boldsymbol{v} \\
\mathbf{0}=\boldsymbol{f}_{2}(\boldsymbol{x}, \boldsymbol{\mu})+\boldsymbol{p}_{2}(\boldsymbol{x}, \boldsymbol{\mu}) \boldsymbol{z}+\boldsymbol{g}_{2}(\boldsymbol{x}, \boldsymbol{\mu}) \boldsymbol{u}+\boldsymbol{q}_{2}(\boldsymbol{x}, \boldsymbol{\mu}) \boldsymbol{v} \\
e=h(\boldsymbol{x})-q_{0}(\boldsymbol{v}, \boldsymbol{\mu})
\end{gathered}
$$

where $\boldsymbol{x} \in \Re^{n}$ and $\boldsymbol{z} \in \Re^{s}$ are the system states, $\boldsymbol{u} \in \Re$ is the control input, $\boldsymbol{\mu} \in U \subset \Re^{m}$ is the plant uncertain parameter vector where $U$ is a compact set, $\boldsymbol{\mu}_{0} \in \Re^{m}$ is the nominal value of $\boldsymbol{\mu}$ which is known. $e \in \Re$ is the system output representing the tracking error. $\boldsymbol{f}_{1}(\boldsymbol{x}, \boldsymbol{\mu}), \boldsymbol{f}_{2}(\boldsymbol{x}, \boldsymbol{\mu}), \boldsymbol{p}_{1}(\boldsymbol{x}, \boldsymbol{\mu}), \boldsymbol{p}_{2}(\boldsymbol{x}, \boldsymbol{\mu}), \boldsymbol{g}_{1}(\boldsymbol{x}, \boldsymbol{\mu})$, $\boldsymbol{g}_{2}(\boldsymbol{x}, \boldsymbol{\mu}), \boldsymbol{q}_{1}(\boldsymbol{x}, \boldsymbol{\mu})$ and $\boldsymbol{q}_{2}(\boldsymbol{x}, \boldsymbol{\mu})$ are matrix-valued functions with dimensions $n \times 1, s \times 1, n \times s, s \times s, n \times 1$, $s \times 1, n \times a$ and $s \times a$, respectively. $\boldsymbol{v} \in \Re^{a}$ is the exogenous signal representing the disturbance and/or the reference input described by

$$
\dot{v}=\boldsymbol{S} \boldsymbol{v}
$$

All functions in (1) are globally defined, sufficiently smooth, and satisfy $\boldsymbol{f}_{1}(\mathbf{0}, \boldsymbol{\mu})=\mathbf{0}, f_{2}(\mathbf{0}, \boldsymbol{\mu})=\mathbf{0}, h(\mathbf{0})=$ $\mathbf{0}$ and $q_{0}(\mathbf{0}, \boldsymbol{\mu})=0$. It is assumed that all the eigenvalues of the matrix $\boldsymbol{S}$ are simple and have zero-real-parts. So for any $\boldsymbol{v}(0) \in \Re^{a}$, there is a compact set $V \in \Re^{a}$ containing the origin of $\Re^{a}$, such that $\boldsymbol{v}(t) \in V$.
Global robust output regulation problem Given a singular nonlinear system (1) and exosystem (2), design a feedback control law

$$
\begin{aligned}
\boldsymbol{u} & =\boldsymbol{u}_{k}(\boldsymbol{x}, \boldsymbol{z}, \boldsymbol{\zeta}, e) \\
\dot{\boldsymbol{\zeta}} & =\boldsymbol{g}_{\zeta}(\boldsymbol{x}, \boldsymbol{z}, \boldsymbol{\zeta}, e)
\end{aligned}
$$

with $\zeta \in \Re^{n_{\zeta}}$ being the state of control law, such that, for any initial states $\boldsymbol{x}(0) \in \Re^{n}, \boldsymbol{z}(0) \in \Re^{s}, \boldsymbol{\zeta}(0) \in \Re^{n_{\zeta}}$, and $\boldsymbol{v}(0) \in \Re^{a}$, the trajectories of the closed-loop system composed of (1), (2) and (3) exist and are bounded for all $t \geqslant 0$, and satisfy $\lim _{t \rightarrow \infty} e(t)=0$.

Remark 1 Most of the existing results on the output regulation problem of nonlinear singular systems are limited to local solutions, e.g., [20,22-24]. This paper aims to establish a global solution for the output regulation problem of singular nonlinear systems. To this target, a class of nonlinear singular system in the form of (1) is addressed in this paper. Though it is in a special form in which the partial state $\boldsymbol{z}$ appears linearly, there exist some physical systems that can be modeled as singular nonlinear systems in the form of (1), e.g. a robot with end effector in contact with a rigid surface [2], a vapor-liquid reaction system [3]. Various control problems are investigated in the literature for the nonlinear singular system in the form of (1), such as, feedback control [4], disturbance decoupling [5], and output tracking [7].

Assumption $1 \quad \operatorname{rank}\left[\boldsymbol{p}_{2}(\boldsymbol{x}, \boldsymbol{\mu}) \quad \boldsymbol{g}_{2}(\boldsymbol{x}, \boldsymbol{\mu})\right]=s$ and there exists a matrix $\gamma(\boldsymbol{x})$ such that $\boldsymbol{p}_{2}(\boldsymbol{x}, \boldsymbol{\mu})+$ $\boldsymbol{g}_{2}(\boldsymbol{x}, \boldsymbol{\mu}) \gamma(\boldsymbol{x})$ is non-singular for all $\boldsymbol{\mu} \in U$.

Remark 2 Assumption 1 is a regularizable condition of the singular nonlinear system (1). It is worth noting that the matrix $\boldsymbol{p}_{2}(x, \mu)$ may be a singular matrix, and we cannot obtain a unique $\boldsymbol{z}$ by implicit function theorem. As shown in [4], Assumption 1 guarantees that there exists a smooth regular feedback in the form of $u=\omega+\gamma(\boldsymbol{x}) \boldsymbol{z}$ such that the closed-loop system has a unique solution for any continuous input $\omega$. Moreover, with Assumption 1, we can define the strong relative degree for the singular nonlinear system (1). In fact, we denote $L_{\boldsymbol{f}_{1}} \boldsymbol{f}_{2}(\boldsymbol{x}, \boldsymbol{\mu})=$ $\frac{\partial \boldsymbol{f}_{2}(\boldsymbol{x}, \boldsymbol{\mu})}{\partial \boldsymbol{x}} \boldsymbol{f}_{1}(\boldsymbol{x}, \boldsymbol{\mu})$ for some smooth functions $\boldsymbol{f}_{1}(\boldsymbol{x}, \boldsymbol{\mu})$ and $\boldsymbol{f}_{2}(\boldsymbol{x}, \boldsymbol{\mu})$, the strong relative degree of the system (1) can be calculated by the following algorithm.

Step 1 Let $\xi_{1}=h(\boldsymbol{x})$ and calculate $L_{\boldsymbol{f}_{1}} \xi_{1}, L_{\boldsymbol{p}_{1}} \xi_{1}$ and $L_{g_{1}} \xi_{1}$. If

$$
\operatorname{rank}\left[\begin{array}{cc}
L_{\boldsymbol{p}_{1}} \xi_{1} & L_{\boldsymbol{g}_{1}} \xi_{1} \\
\boldsymbol{p}_{2}(\boldsymbol{x}, \boldsymbol{\mu}) & \boldsymbol{g}_{2}(\boldsymbol{x}, \boldsymbol{\mu})
\end{array}\right]=s
$$

then there exists a vector-valued function $\boldsymbol{E}_{1}(\boldsymbol{x}, \boldsymbol{\mu}) \in$ $\Re^{1 \times s}$ such that

$$
\left[\begin{array}{ll}
L_{\boldsymbol{p}_{1}} \xi_{1} & L_{\boldsymbol{g}_{1}} \xi_{1}
\end{array}\right]=\boldsymbol{E}_{1}(\boldsymbol{x}, \boldsymbol{\mu})\left[\begin{array}{ll}
\boldsymbol{p}_{2}(\boldsymbol{x}, \boldsymbol{\mu}) & \left.\boldsymbol{g}_{2}(\boldsymbol{x}, \boldsymbol{\mu})\right]
\end{array}\right.
$$


Denote $\hat{\xi}_{2}(\boldsymbol{x}, \boldsymbol{\mu})=L_{\boldsymbol{f}_{1}} \xi_{1}-\boldsymbol{E}_{1}(\boldsymbol{x}, \boldsymbol{\mu}) \boldsymbol{f}_{2}$ and $\xi_{2}\left(\boldsymbol{x}, \boldsymbol{\mu}_{0}\right)=$ $\hat{\xi}_{2}\left(\boldsymbol{x}, \boldsymbol{\mu}_{0}\right)$; Otherwise, let $\rho=1$ and end the algorithm.

Step $\mathbf{k}$ We assume that a sequence of $\xi_{1}\left(\boldsymbol{x}, \boldsymbol{\mu}_{0}\right), \ldots$, $\xi_{k}\left(\boldsymbol{x}, \boldsymbol{\mu}_{0}\right)$ is defined. Calculate $L_{\boldsymbol{f}_{1}} \xi_{k}, L_{\boldsymbol{p}_{1}} \xi_{k}$ and $L_{\boldsymbol{g}_{1}} \xi_{k}$. If

$$
\operatorname{rank}\left[\begin{array}{cc}
L_{\boldsymbol{p}_{1}} \xi_{k} & L_{\boldsymbol{g}_{1}} \xi_{k} \\
\boldsymbol{p}_{2}(\boldsymbol{x}, \boldsymbol{\mu}) & \boldsymbol{g}_{2}(\boldsymbol{x}, \boldsymbol{\mu})
\end{array}\right]=s
$$

then there exists a vector-valued function $E_{k}\left(\boldsymbol{x}, \boldsymbol{\mu}, \mu_{0}\right) \in$ $\Re^{1 \times s}$ such that

$\left[\begin{array}{ll}L_{\boldsymbol{p}_{1}} \xi_{k} & L_{\boldsymbol{g}_{1}} \xi_{k}\end{array}\right]=E_{k}\left(\boldsymbol{x}, \boldsymbol{\mu}, \mu_{0}\right)\left[\begin{array}{ll}\boldsymbol{p}_{2}(\boldsymbol{x}, \boldsymbol{\mu}) & \left.\boldsymbol{g}_{2}(\boldsymbol{x}, \boldsymbol{\mu})\right]\end{array}\right.$

Denote $\hat{\xi}_{k+1}\left(\boldsymbol{x}, \boldsymbol{\mu}, \boldsymbol{\mu}_{0}\right)=L_{\boldsymbol{f}_{1}} \xi_{k}-\boldsymbol{E}_{k}\left(\boldsymbol{x}, \boldsymbol{\mu}, \boldsymbol{\mu}_{0}\right) \boldsymbol{f}_{2}$ and $\xi_{k+1}\left(\boldsymbol{x}, \boldsymbol{\mu}_{0}\right)=\hat{\xi}_{k+1}\left(\boldsymbol{x}, \boldsymbol{\mu}_{0}, \boldsymbol{\mu}_{0}\right)$; otherwise, let $\rho=k$ and end the algorithm.

The $\rho$ obtained in the above algorithm is called strong relative degree of the system (1). Similar algorithms have been used to develop the state-space description for differential-algebraic equation systems in [2] and [4]. It is worth mentioning that the strong relative degree of the system (1) may be different with the integer $k$ of the system (1) with $\boldsymbol{\mu}=\boldsymbol{\mu}_{0}$ by the Algorithm 1 in [4], because $\xi_{k}\left(\boldsymbol{x}, \boldsymbol{\mu}_{0}\right)(k=2, \ldots, \rho)$ is defined recursively and independent of $\boldsymbol{\mu}$. Please refer to [4] for more details on the algorithm.

Assumption 2 The system (1) has a strong relative degree $\rho \leqslant n$ for all $\boldsymbol{\mu} \in U$.

\section{Problem conversion}

Now, based on the assumption that the singular nonlinear system has a strong relative degree, we can show that a global diffeomorphism map can be constructed to transfer the singular nonlinear system into a new singular nonlinear system with a special structure.

Lemma 1 For system (1), under Assumptions 1 and 2, there exists a smooth function $\boldsymbol{\eta}\left(\boldsymbol{x}, \boldsymbol{\mu}_{0}\right)$, such that

$$
T\left(\boldsymbol{x}, \boldsymbol{\mu}_{0}\right)=\left[\begin{array}{c}
\boldsymbol{\eta}\left(\boldsymbol{x}, \boldsymbol{\mu}_{0}\right) \\
\xi_{1}\left(\boldsymbol{x}, \boldsymbol{\mu}_{0}\right) \\
\vdots \\
\xi_{\rho}\left(\boldsymbol{x}, \boldsymbol{\mu}_{0}\right)
\end{array}\right], \boldsymbol{\eta}\left(\boldsymbol{x}, \boldsymbol{\mu}_{0}\right) \in \Re^{n-\rho}
$$

is a global diffeomorphism and

$$
L_{\boldsymbol{g}(\boldsymbol{x}, \boldsymbol{\mu})} \boldsymbol{\eta}\left(\boldsymbol{x}, \boldsymbol{\mu}_{0}\right)=0
$$

where

$$
\boldsymbol{g}(\boldsymbol{x}, \boldsymbol{\mu})=\boldsymbol{g}_{1}(\boldsymbol{x}, \boldsymbol{\mu})-\boldsymbol{\kappa}_{1}(\boldsymbol{x}, \boldsymbol{\mu}) \boldsymbol{\kappa}_{2}^{-1}(\boldsymbol{x}, \boldsymbol{\mu}) \boldsymbol{g}_{2}(\boldsymbol{x}, \boldsymbol{\mu})
$$

and

$$
\begin{aligned}
\boldsymbol{\kappa}_{1}(\boldsymbol{x}, \boldsymbol{\mu}) & =\boldsymbol{p}_{1}(\boldsymbol{x}, \boldsymbol{\mu})+\boldsymbol{g}_{1}(\boldsymbol{x}, \boldsymbol{\mu}) \gamma(\boldsymbol{x}) \\
\boldsymbol{\kappa}_{2}(\boldsymbol{x}, \boldsymbol{\mu}) & =\boldsymbol{p}_{2}(\boldsymbol{x}, \boldsymbol{\mu})+\boldsymbol{g}_{2}(\boldsymbol{x}, \boldsymbol{\mu}) \gamma(\boldsymbol{x})
\end{aligned}
$$

Proof Let $u=w+\gamma(\boldsymbol{x}) \boldsymbol{z}$, the system (1) can be written as

$$
\begin{gathered}
\dot{\boldsymbol{x}}=\boldsymbol{f}_{1}(\boldsymbol{x}, \boldsymbol{\mu})+\boldsymbol{g}_{1}(\boldsymbol{x}, \boldsymbol{\mu}) w+\boldsymbol{\kappa}_{1}(\boldsymbol{x}, \boldsymbol{\mu}) \boldsymbol{z}+\boldsymbol{q}_{1}(\boldsymbol{x}, \boldsymbol{\mu}) \boldsymbol{v} \\
\underline{0}=\boldsymbol{f}_{2}(\boldsymbol{x}, \boldsymbol{\mu})+\boldsymbol{g}_{2}(\boldsymbol{x}, \boldsymbol{\mu}) w+\boldsymbol{\kappa}_{2}(\boldsymbol{x}, \boldsymbol{\mu}) \boldsymbol{z}+\boldsymbol{q}_{2}(\boldsymbol{x}, \boldsymbol{\mu}) \boldsymbol{v} \\
e=h(\boldsymbol{x})-q_{0}(\boldsymbol{v}, \boldsymbol{\mu})
\end{gathered}
$$

where $\boldsymbol{\kappa}_{1}(\boldsymbol{x}, \boldsymbol{\mu})$ and $\boldsymbol{\kappa}_{2}(\boldsymbol{x}, \boldsymbol{\mu})$ are defined by (6) and (7) respectively. Under Assumption $1, \boldsymbol{\kappa}_{2}(\boldsymbol{x}, \boldsymbol{\mu})$ is nonsingular, thus the variable $z$ can be uniquely determined by

$$
\boldsymbol{z}=-\boldsymbol{\kappa}_{2}^{-1}(\boldsymbol{x}, \boldsymbol{\mu})\left(\boldsymbol{f}_{2}(\boldsymbol{x}, \boldsymbol{\mu})+\boldsymbol{g}_{2}(\boldsymbol{x}, \boldsymbol{\mu}) w+\boldsymbol{q}_{2}(\boldsymbol{x}, \boldsymbol{\mu}) \boldsymbol{v}\right) .
$$

It follows that

$$
\begin{gathered}
\dot{\boldsymbol{x}}=f(\boldsymbol{x}, \boldsymbol{\mu})+\boldsymbol{g}(\boldsymbol{x}, \boldsymbol{\mu}) w+\boldsymbol{q}(\boldsymbol{x}, \boldsymbol{\mu}) \boldsymbol{v} \\
e=h(\boldsymbol{x})-q_{0}(\boldsymbol{v}, \boldsymbol{\mu})
\end{gathered}
$$

where

$$
\begin{gathered}
\boldsymbol{f}(\boldsymbol{x}, \boldsymbol{\mu})=\boldsymbol{f}_{1}(\boldsymbol{x}, \boldsymbol{\mu})-\boldsymbol{\kappa}_{1}(\boldsymbol{x}, \boldsymbol{\mu}) \boldsymbol{\kappa}_{2}^{-1}(\boldsymbol{x}, \boldsymbol{\mu}) \boldsymbol{f}_{2}(\boldsymbol{x}, \boldsymbol{\mu}) \\
\boldsymbol{g}(\boldsymbol{x}, \boldsymbol{\mu})=\boldsymbol{g}_{1}(\boldsymbol{x}, \boldsymbol{\mu})-\boldsymbol{\kappa}_{1}(\boldsymbol{x}, \boldsymbol{\mu}) \boldsymbol{\kappa}_{2}^{-1}(\boldsymbol{x}, \boldsymbol{\mu}) \boldsymbol{g}_{2}(\boldsymbol{x}, \boldsymbol{\mu}) \\
\boldsymbol{q}(\boldsymbol{x}, \boldsymbol{\mu})=\boldsymbol{q}_{1}(\boldsymbol{x}, \boldsymbol{\mu})-\boldsymbol{\kappa}_{1}(\boldsymbol{x}, \boldsymbol{\mu}) \boldsymbol{\kappa}_{2}^{-1}(\boldsymbol{x}, \boldsymbol{\mu}) \boldsymbol{q}_{2}(\boldsymbol{x}, \boldsymbol{\mu})
\end{gathered}
$$

Let $\boldsymbol{q}^{*}\left(\boldsymbol{x}, \boldsymbol{\mu}, \boldsymbol{\mu}_{0}, \boldsymbol{v}\right)=\boldsymbol{q}(\boldsymbol{x}, \boldsymbol{\mu}) \boldsymbol{v}+\boldsymbol{f}(\boldsymbol{x}, \boldsymbol{\mu})-\boldsymbol{f}\left(\boldsymbol{x}, \boldsymbol{\mu}_{0}\right)$, then we have

$$
\begin{gathered}
\dot{\boldsymbol{x}}=\boldsymbol{f}\left(\boldsymbol{x}, \boldsymbol{\mu}_{0}\right)+\boldsymbol{g}(\boldsymbol{x}, \boldsymbol{\mu}) w+\boldsymbol{q}^{*}\left(\boldsymbol{x}, \boldsymbol{\mu}, \boldsymbol{\mu}_{0}, \boldsymbol{v}\right) \\
e=h(\boldsymbol{x})-q_{0}(\boldsymbol{v}, \boldsymbol{\mu}) .
\end{gathered}
$$

With Assumption 2 and Remark 2, there exists $\boldsymbol{E}_{k}\left(\boldsymbol{x}, \boldsymbol{\mu}, \boldsymbol{\mu}_{0}\right)$, for $k=1, \ldots, \rho-1$, such that

$$
\left[\begin{array}{ll}
L_{\boldsymbol{p}_{1}} \xi_{k} & L_{\boldsymbol{g}_{1}} \xi_{k}
\end{array}\right]\left[\begin{array}{cc}
\boldsymbol{I} & 0 \\
\gamma(x) & \boldsymbol{I}
\end{array}\right]=
$$

$$
\boldsymbol{E}_{k}\left(\boldsymbol{x}, \boldsymbol{\mu}, \boldsymbol{\mu}_{0}\right)\left[\begin{array}{ll}
\boldsymbol{p}_{2}(\boldsymbol{x}, \boldsymbol{\mu}) & \boldsymbol{g}_{2}(\boldsymbol{x}, \boldsymbol{\mu})
\end{array}\right]\left[\begin{array}{cc}
\boldsymbol{I} & 0 \\
\gamma(\boldsymbol{x}) & \boldsymbol{I}
\end{array}\right]
$$

which implies

$$
\begin{gathered}
{\left[\begin{array}{ll}
L_{\boldsymbol{p}_{1}} \xi_{k}+L_{\boldsymbol{g}_{1}} \xi_{k} \gamma(\boldsymbol{x}) & L_{\boldsymbol{g}_{1}} \xi_{k}
\end{array}\right]=} \\
\boldsymbol{E}_{k}\left(\boldsymbol{x}, \boldsymbol{\mu}, \boldsymbol{\mu}_{0}\right)\left[\begin{array}{ll}
\boldsymbol{p}_{2}(\boldsymbol{x}, \boldsymbol{\mu})+\boldsymbol{g}_{2}(\boldsymbol{x}, \boldsymbol{\mu}) \boldsymbol{\gamma}(\boldsymbol{x}) & \left.\boldsymbol{g}_{2}(\boldsymbol{x}, \boldsymbol{\mu})\right]
\end{array} .\right.
\end{gathered}
$$

Hence, for $k=1, \ldots, \rho-1$,

$$
\begin{gathered}
\boldsymbol{E}_{k}\left(\boldsymbol{x}, \boldsymbol{\mu}, \boldsymbol{\mu}_{0}\right)=\frac{\partial \xi_{k}}{\partial x} \boldsymbol{\kappa}_{1}(\boldsymbol{x}, \boldsymbol{\mu}) \boldsymbol{\kappa}_{2}^{-1}(\boldsymbol{x}, \boldsymbol{\mu}) \\
L_{\boldsymbol{g}_{1}} \xi_{k}=\boldsymbol{E}_{k}\left(\boldsymbol{x}, \boldsymbol{\mu}, \boldsymbol{\mu}_{0}\right) \boldsymbol{g}_{2}(\boldsymbol{x}, \boldsymbol{\mu}) .
\end{gathered}
$$

It follows that, for $k=1, \ldots, \rho-1$,

$$
L_{\boldsymbol{f}\left(\boldsymbol{x}, \boldsymbol{\mu}_{0}\right)} \xi_{k}\left(\boldsymbol{x}, \boldsymbol{\mu}_{0}\right)=
$$

$$
\left[L_{\boldsymbol{f}_{1}(\boldsymbol{x}, \boldsymbol{\mu})} \xi_{k}-\frac{\partial \xi_{k}}{\partial \boldsymbol{x}} \boldsymbol{\kappa}_{1}(\boldsymbol{x}, \boldsymbol{\mu}) \boldsymbol{\kappa}_{2}^{-1}(\boldsymbol{x}, \boldsymbol{\mu}) \boldsymbol{f}_{2}(\boldsymbol{x}, \boldsymbol{\mu})\right]_{\boldsymbol{\mu}=\boldsymbol{\mu}_{0}}=
$$




$$
\begin{gathered}
{\left[L_{\boldsymbol{f}_{1}(\boldsymbol{x}, \boldsymbol{\mu})} \xi_{k}-\boldsymbol{E}_{k}\left(\boldsymbol{x}, \boldsymbol{\mu}, \boldsymbol{\mu}_{0}\right) \boldsymbol{f}_{2}(\boldsymbol{x}, \boldsymbol{\mu})\right]_{\boldsymbol{\mu}=\boldsymbol{\mu}_{0}}=} \\
\xi_{k+1}\left(\boldsymbol{x}, \boldsymbol{\mu}_{0}\right) \\
L_{\boldsymbol{g}(\boldsymbol{x}, \boldsymbol{\mu})} \xi_{k}\left(\boldsymbol{x}, \boldsymbol{\mu}_{0}\right)= \\
\frac{\partial \xi_{k}}{\partial \boldsymbol{x}}\left[\boldsymbol{g}_{1}(\boldsymbol{x}, \boldsymbol{\mu})-\kappa_{1}(\boldsymbol{x}, \boldsymbol{\mu}) \boldsymbol{\kappa}_{2}^{-1}(\boldsymbol{x}, \boldsymbol{\mu}) \boldsymbol{g}_{2}(\boldsymbol{x}, \boldsymbol{\mu})\right]= \\
L_{\boldsymbol{g}_{1}} \xi_{k}-\boldsymbol{E}_{k}\left(\boldsymbol{x}, \boldsymbol{\mu}, \boldsymbol{\mu}_{0}\right) \boldsymbol{g}_{2}(\boldsymbol{x}, \boldsymbol{\mu})=0 .
\end{gathered}
$$

and

$$
L_{\boldsymbol{g}} \xi_{\rho}=L_{\boldsymbol{g}_{1}} \xi_{\rho}-\frac{\partial \xi_{\rho}}{\partial \boldsymbol{x}} \boldsymbol{\kappa}_{1}(\boldsymbol{x}, \boldsymbol{\mu}) \boldsymbol{\kappa}_{2}^{-1}(\boldsymbol{x}, \boldsymbol{\mu}) \boldsymbol{g}_{2}(\boldsymbol{x}, \boldsymbol{\mu}) \neq 0
$$

Considering the system (8), and viewing $\boldsymbol{q}^{*}\left(\boldsymbol{x}, \boldsymbol{\mu}, \boldsymbol{\mu}_{0}, \boldsymbol{v}\right)$ as the perturbation, (9), (10) and (11) imply that the system (8) has a relative degree $\rho$. By Theorem 13.1 in [26] (or Proposition 4.1.3 in [27]), the map

$$
T\left(\boldsymbol{x}, \boldsymbol{\mu}_{0}\right)=\left[\begin{array}{c}
\eta\left(\boldsymbol{x}, \boldsymbol{\mu}_{0}\right) \\
\xi_{1}\left(\boldsymbol{x}, \boldsymbol{\mu}_{0}\right) \\
\vdots \\
\xi_{\rho}\left(\boldsymbol{x}, \boldsymbol{\mu}_{0}\right)
\end{array}\right], \boldsymbol{\eta} \in \Re^{n-\rho}
$$

is a global diffeomorphism such that

$$
L_{\boldsymbol{g}(\boldsymbol{x}, \boldsymbol{\mu})} \boldsymbol{\eta}\left(\boldsymbol{x}, \boldsymbol{\mu}_{0}\right)=0
$$

which completes the proof of Lemma 1.

With Lemma 1 and the algebra equation of (1), it can be verified that, for $i=1, \ldots, \rho-1$,

$$
\begin{gathered}
\dot{\xi}_{i}=\frac{\partial \xi_{i}}{\partial \boldsymbol{x}}\left(\boldsymbol{f}_{1}(\boldsymbol{x}, \boldsymbol{\mu})+\boldsymbol{p}_{1}(\boldsymbol{x}, \boldsymbol{\mu}) z+\boldsymbol{g}_{1}(\boldsymbol{x}, \boldsymbol{\mu}) \boldsymbol{u}+\boldsymbol{q}_{1}(\boldsymbol{x}, \boldsymbol{\mu}) \boldsymbol{v}\right)= \\
\frac{\partial \xi_{i}}{\partial \boldsymbol{x}}\left(\boldsymbol{f}_{1}(\boldsymbol{x}, \boldsymbol{\mu})+\boldsymbol{q}_{1}(\boldsymbol{x}, \boldsymbol{\mu}) \boldsymbol{v}\right)+ \\
\boldsymbol{E}_{i}\left(\boldsymbol{x}, \boldsymbol{\mu}, \boldsymbol{\mu}_{0}\right)\left(\boldsymbol{p}_{2}(\boldsymbol{x}, \boldsymbol{\mu}) \boldsymbol{z}+\boldsymbol{g}_{2}(\boldsymbol{x}, \boldsymbol{\mu}) \boldsymbol{u}\right)= \\
\frac{\partial \xi_{i}}{\partial x}\left(\boldsymbol{f}_{1}(\boldsymbol{x}, \boldsymbol{\mu})+\boldsymbol{q}_{1}(\boldsymbol{x}, \boldsymbol{\mu}) \boldsymbol{v}\right)- \\
\boldsymbol{E}_{i}\left(\boldsymbol{x}, \boldsymbol{\mu}, \boldsymbol{\mu}_{0}\right)\left(\boldsymbol{f}_{2}(\boldsymbol{x}, \boldsymbol{\mu})+\boldsymbol{q}_{2}(\boldsymbol{x}, \boldsymbol{\mu}) \boldsymbol{v}\right)= \\
\xi_{i+1}+\varphi_{i}\left(\boldsymbol{\eta}, \xi_{1}, \xi_{2}, \ldots, \xi_{\rho}, \boldsymbol{v}, \boldsymbol{\mu}, \boldsymbol{\mu}_{0}\right)
\end{gathered}
$$

where

$$
\begin{gathered}
\varphi_{i}\left(\boldsymbol{\eta}, \xi_{1}, \xi_{2}, \ldots, \xi_{\rho}, \boldsymbol{v}, \boldsymbol{\mu}, \boldsymbol{\mu}_{0}\right)=\frac{\partial \xi_{k}}{\partial x}\left(\boldsymbol{f}_{1}(\boldsymbol{x}, \boldsymbol{\mu})-\right. \\
\left.\boldsymbol{f}_{1}\left(\boldsymbol{x}, \boldsymbol{\mu}_{0}\right)+\boldsymbol{q}_{1}(\boldsymbol{x}, \boldsymbol{\mu}) \boldsymbol{v}\right)-\boldsymbol{E}_{i}\left(\boldsymbol{x}, \boldsymbol{\mu}, \boldsymbol{\mu}_{0}\right)\left(\boldsymbol{f}_{2}(\boldsymbol{x}, \boldsymbol{\mu})-\right. \\
\left.\boldsymbol{f}_{2}\left(\boldsymbol{x}, \boldsymbol{\mu}_{0}\right)+\boldsymbol{q}_{2}(\boldsymbol{x}, \boldsymbol{\mu}) \boldsymbol{v}\right) .
\end{gathered}
$$

Let $\boldsymbol{E}_{0}\left(\boldsymbol{x}, \boldsymbol{\mu}, \boldsymbol{\mu}_{0}\right)=\frac{\partial \eta}{\partial \boldsymbol{x}} \boldsymbol{\kappa}_{1}(\boldsymbol{x}, \boldsymbol{\mu}) \boldsymbol{\kappa}_{2}^{-1}(\boldsymbol{x}, \boldsymbol{\mu})$, it follows that

$$
\boldsymbol{E}_{0}\left(\boldsymbol{x}, \boldsymbol{\mu}, \boldsymbol{\mu}_{0}\right) \boldsymbol{\kappa}_{2}(\boldsymbol{x}, \boldsymbol{\mu})=\frac{\partial \eta}{\partial \boldsymbol{x}} \boldsymbol{\kappa}_{1}(\boldsymbol{x}, \boldsymbol{\mu}),
$$

then

$$
\begin{gathered}
\boldsymbol{E}_{0}\left(\boldsymbol{x}, \boldsymbol{\mu}, \boldsymbol{\mu}_{0}\right)\left(\boldsymbol{p}_{2}(\boldsymbol{x}, \boldsymbol{\mu})+\boldsymbol{g}_{2}(\boldsymbol{x}, \boldsymbol{\mu}) \gamma(\boldsymbol{x})\right)= \\
\frac{\partial \eta}{\partial \boldsymbol{x}}\left(\boldsymbol{p}_{1}(\boldsymbol{x}, \boldsymbol{\mu})+\boldsymbol{g}_{1}(\boldsymbol{x}, \boldsymbol{\mu}) \gamma(\boldsymbol{x})\right) .
\end{gathered}
$$

By (4), we have

$$
\begin{gathered}
\frac{\partial \eta}{\partial \boldsymbol{x}} \boldsymbol{g}_{1}(\boldsymbol{x}, \boldsymbol{\mu})=\frac{\partial \eta}{\partial \boldsymbol{x}} \boldsymbol{\kappa}_{1}(\boldsymbol{x}, \boldsymbol{\mu}) \boldsymbol{\kappa}_{2}^{-1}(\boldsymbol{x}, \boldsymbol{\mu}) \boldsymbol{g}_{2}(\boldsymbol{x}, \boldsymbol{\mu})= \\
\boldsymbol{E}_{0}\left(\boldsymbol{x}, \boldsymbol{\mu}, \boldsymbol{\mu}_{0}\right) \boldsymbol{g}_{2}(\boldsymbol{x}, \boldsymbol{\mu}) .
\end{gathered}
$$

Substituting (14) into (13) yields

$$
\frac{\partial \boldsymbol{\eta}}{\partial \boldsymbol{x}} \boldsymbol{p}_{1}(\boldsymbol{x}, \boldsymbol{\mu})=\boldsymbol{E}_{0}\left(\boldsymbol{x}, \boldsymbol{\mu}, \boldsymbol{\mu}_{0}\right) \boldsymbol{p}_{2}(\boldsymbol{x}, \boldsymbol{\mu})
$$

Furthermore, (14) and (15) can be rewritten as

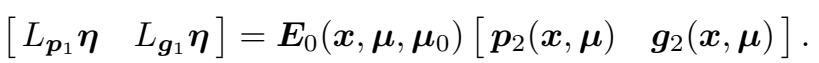

Thus, with the algebra equation of (1), we have

$$
\begin{gathered}
\dot{\boldsymbol{\eta}}=\frac{\partial \eta}{\partial \boldsymbol{x}}\left(\boldsymbol{f}_{1}(\boldsymbol{x}, \boldsymbol{\mu})+\boldsymbol{p}_{1}(\boldsymbol{x}, \boldsymbol{\mu}) \boldsymbol{z}+\boldsymbol{g}_{1}(\boldsymbol{x}, \boldsymbol{\mu}) \boldsymbol{u}+\boldsymbol{q}_{1}(\boldsymbol{x}, \boldsymbol{\mu}) \boldsymbol{v}\right)= \\
\frac{\partial \boldsymbol{\eta}}{\partial \boldsymbol{x}}\left(\boldsymbol{f}_{1}(\boldsymbol{x}, \boldsymbol{\mu})+\boldsymbol{q}_{1}(\boldsymbol{x}, \boldsymbol{\mu}) \boldsymbol{v}\right)+ \\
\boldsymbol{E}_{0}\left(\boldsymbol{x}, \boldsymbol{\mu}, \boldsymbol{\mu}_{0}\right)\left(\boldsymbol{p}_{2}(\boldsymbol{x}, \boldsymbol{\mu}) \boldsymbol{z}+\boldsymbol{g}_{2}(\boldsymbol{x}, \boldsymbol{\mu}) \boldsymbol{u}\right)= \\
\frac{\partial \boldsymbol{\eta}}{\partial \boldsymbol{x}}\left(\boldsymbol{f}_{1}(\boldsymbol{x}, \boldsymbol{\mu})+\boldsymbol{q}_{1}(\boldsymbol{x}, \boldsymbol{\mu}) \boldsymbol{v}\right)- \\
\boldsymbol{E}_{0}\left(\boldsymbol{x}, \boldsymbol{\mu}, \boldsymbol{\mu}_{0}\right)\left(\boldsymbol{f}_{2}(\boldsymbol{x}, \boldsymbol{\mu})+\boldsymbol{q}_{2}(\boldsymbol{x}, \boldsymbol{\mu}) \boldsymbol{v}\right)
\end{gathered}
$$

By (12) and (16), it is clear that the global diffeomorphism map $T\left(\boldsymbol{x}, \boldsymbol{\mu}_{0}\right)$ transfers the system (1) into the following form:

$$
\begin{gathered}
\dot{\boldsymbol{\eta}}=\boldsymbol{\psi}\left(\boldsymbol{\eta}, \xi_{1}, \xi_{2}, \ldots, \xi_{\rho}, \boldsymbol{v}, \boldsymbol{\mu}, \boldsymbol{\mu}_{0}\right) \\
\dot{\xi}_{i}=\xi_{i+1}+\varphi_{i}\left(\boldsymbol{\eta}, \xi_{1}, \xi_{2}, \ldots, \xi_{\rho}, \boldsymbol{v}, \boldsymbol{\mu}, \boldsymbol{\mu}_{0}\right) \\
\dot{\xi}_{\rho}=\boldsymbol{f}_{3}\left(\boldsymbol{\eta}, \boldsymbol{\xi}, \boldsymbol{\mu}, \boldsymbol{\mu}_{0}\right)+\boldsymbol{p}_{3}\left(\boldsymbol{\eta}, \boldsymbol{\xi}, \boldsymbol{\mu}, \boldsymbol{\mu}_{0}\right) \boldsymbol{z}+ \\
\boldsymbol{g}_{3}\left(\boldsymbol{\eta}, \boldsymbol{\xi}, \boldsymbol{\mu}, \boldsymbol{\mu}_{0}\right) \boldsymbol{u}+\boldsymbol{q}_{3}\left(\boldsymbol{\eta}, \boldsymbol{\xi}, \boldsymbol{\mu}, \boldsymbol{\mu}_{0}\right) \boldsymbol{v} \\
0=\boldsymbol{f}_{2}^{\prime}\left(\boldsymbol{\eta}, \boldsymbol{\xi}, \boldsymbol{\mu}, \boldsymbol{\mu}_{0}\right)+\boldsymbol{p}_{2}^{\prime}\left(\boldsymbol{\eta}, \boldsymbol{\xi}, \boldsymbol{\mu}, \boldsymbol{\mu}_{0}\right) \boldsymbol{z}+ \\
\boldsymbol{g}_{2}^{\prime}\left(\boldsymbol{\eta}, \boldsymbol{\xi}, \boldsymbol{\mu}, \boldsymbol{\mu}_{0}\right) \boldsymbol{u}+\boldsymbol{q}_{2}^{\prime}\left(\boldsymbol{\eta}, \boldsymbol{\xi}, \boldsymbol{\mu}, \boldsymbol{\mu}_{0}\right) \boldsymbol{v} \\
e=\xi_{1}-\boldsymbol{q}_{0}(\boldsymbol{v}, \boldsymbol{\mu})
\end{gathered}
$$

where $i=1, \ldots, \rho-1, \xi=\operatorname{col}\left(\xi_{1}, \ldots, \xi_{\rho}\right)$,

$$
\begin{gathered}
\boldsymbol{\psi}\left(\boldsymbol{\eta}, \xi_{1}, \xi_{2}, \ldots, \xi_{\rho}, \boldsymbol{v}, \boldsymbol{\mu}, \boldsymbol{\mu}_{0}\right)= \\
\frac{\partial \boldsymbol{\eta}}{\partial \boldsymbol{x}}\left(\boldsymbol{f}_{1}(\boldsymbol{x}, \boldsymbol{\mu})+\boldsymbol{q}_{1}(\boldsymbol{\eta}, \boldsymbol{\xi}, \boldsymbol{\mu}) \boldsymbol{v}\right)- \\
\boldsymbol{E}_{0}\left(\boldsymbol{x}, \boldsymbol{\mu}, \boldsymbol{\mu}_{0}\right)\left(\boldsymbol{f}_{2}(\boldsymbol{x}, \boldsymbol{\mu})+\boldsymbol{q}_{2}(\boldsymbol{x}, \boldsymbol{\mu}) \boldsymbol{v}\right)
\end{gathered}
$$

and

$$
\begin{gathered}
\boldsymbol{f}_{3}\left(\boldsymbol{\eta}, \boldsymbol{\xi}, \boldsymbol{\mu}, \boldsymbol{\mu}_{0}\right)=\frac{\partial \xi_{\rho}}{\partial \boldsymbol{x}} \boldsymbol{f}_{1}(\boldsymbol{x}, \boldsymbol{\mu}) \\
\boldsymbol{f}_{2}^{\prime}\left(\boldsymbol{\eta}, \boldsymbol{\xi}, \boldsymbol{\mu}, \boldsymbol{\mu}_{0}\right)=\boldsymbol{f}_{2}(\boldsymbol{x}, \boldsymbol{\mu}) \\
\boldsymbol{p}_{3}\left(\boldsymbol{\eta}, \boldsymbol{\xi}, \boldsymbol{\mu}, \boldsymbol{\mu}_{0}\right)=\frac{\partial \xi_{\rho}}{\partial \boldsymbol{x}} \boldsymbol{p}_{1}(\boldsymbol{x}, \boldsymbol{\mu})
\end{gathered}
$$




$$
\begin{gathered}
\boldsymbol{p}_{2}^{\prime}\left(\boldsymbol{\eta}, \boldsymbol{\xi}, \boldsymbol{\mu}, \boldsymbol{\mu}_{0}\right)=\boldsymbol{p}_{2}(\boldsymbol{x}, \boldsymbol{\mu}) \\
\boldsymbol{g}_{3}\left(\boldsymbol{\eta}, \boldsymbol{\xi}, \boldsymbol{\mu}, \boldsymbol{\mu}_{0}\right)=\frac{\partial \xi_{\rho}}{\partial \boldsymbol{x}} \boldsymbol{g}_{1}(\boldsymbol{x}, \boldsymbol{\mu}) \\
\boldsymbol{g}_{2}^{\prime}\left(\boldsymbol{\eta}, \boldsymbol{\xi}, \boldsymbol{\mu}, \boldsymbol{\mu}_{0}\right)=\boldsymbol{g}_{2}(\boldsymbol{x}, \boldsymbol{\mu}) \\
\boldsymbol{q}_{3}\left(\boldsymbol{\eta}, \boldsymbol{\xi}, \boldsymbol{\mu}, \boldsymbol{\mu}_{0}\right)=\frac{\partial \xi_{\rho}}{\partial \boldsymbol{x}} \boldsymbol{q}_{1}(\boldsymbol{x}, \boldsymbol{\mu}) \\
\boldsymbol{q}_{2}^{\prime}\left(\boldsymbol{\eta}, \boldsymbol{\xi}, \boldsymbol{\mu}, \boldsymbol{\mu}_{0}\right)=\boldsymbol{q}_{2}(\boldsymbol{x}, \boldsymbol{\mu}) .
\end{gathered}
$$

To deal with the global robust output regulation problem for singular nonlinear system (17), we need two assumptions as follows.

Assumption 3 For the system (17), there exist sufficiently smooth functions $\boldsymbol{\alpha}(\boldsymbol{v}, \boldsymbol{\mu}) \in \Re^{n-\rho}, \boldsymbol{\beta}(\boldsymbol{v}, \boldsymbol{\mu})=$ $\operatorname{col}\left(\beta_{1}(v, \mu), \ldots, \beta_{\rho}(v, \mu)\right) \in \Re^{\rho}, \boldsymbol{z}(\boldsymbol{v}, \boldsymbol{\mu}) \in \Re^{s}$, $\boldsymbol{u}(\boldsymbol{v}, \boldsymbol{\mu}) \in \Re$ with $\boldsymbol{\alpha}(0,0)=\mathbf{0}, \beta(0,0)=\mathbf{0}, \boldsymbol{z}(0,0)=\mathbf{0}$ and $\boldsymbol{u}(0,0)=\mathbf{0}$, such that

$$
\begin{gathered}
\frac{\partial \boldsymbol{\alpha}}{\partial \boldsymbol{v}} \boldsymbol{S} \boldsymbol{v}=\boldsymbol{\psi}\left(\boldsymbol{\alpha}, \beta_{1}, \beta_{2}, \ldots, \beta_{\rho}, \boldsymbol{v}, \boldsymbol{\mu}, \boldsymbol{\mu}_{0}\right) \\
\frac{\partial \beta_{i}}{\partial \boldsymbol{v}} \boldsymbol{S} \boldsymbol{v}=\beta_{i+1}+\varphi_{i}\left(\boldsymbol{\alpha}, \beta_{1}, \beta_{2}, \ldots, \beta_{\rho}, \boldsymbol{v}, \boldsymbol{\mu}, \boldsymbol{\mu}_{0}\right) \\
\frac{\partial \beta_{\rho}}{\partial \boldsymbol{v}} \boldsymbol{S} \boldsymbol{v}=\boldsymbol{f}_{3}\left(\boldsymbol{\alpha}, \boldsymbol{\beta}, \boldsymbol{\mu}, \boldsymbol{\mu}_{0}\right)+\boldsymbol{p}_{3}\left(\boldsymbol{\alpha}, \boldsymbol{\beta}, \boldsymbol{\mu}, \boldsymbol{\mu}_{0}\right) \boldsymbol{z}+ \\
\boldsymbol{g}_{3}\left(\boldsymbol{\alpha}, \boldsymbol{\beta}, \boldsymbol{\mu}, \boldsymbol{\mu}_{0}\right) \boldsymbol{u}+\boldsymbol{q}_{3}\left(\boldsymbol{\alpha}, \boldsymbol{\beta}, \boldsymbol{\mu}, \boldsymbol{\mu}_{0}\right) \boldsymbol{v} \\
\mathbf{0}=\boldsymbol{f}_{2}^{\prime}\left(\boldsymbol{\alpha}, \boldsymbol{\beta}, \boldsymbol{\mu}, \boldsymbol{\mu}_{0}\right)+\boldsymbol{p}_{2}^{\prime}\left(\boldsymbol{\alpha}, \boldsymbol{\beta}, \boldsymbol{\mu}, \boldsymbol{\mu}_{0}\right) \boldsymbol{z}+ \\
\boldsymbol{g}_{2}^{\prime}\left(\boldsymbol{\alpha}, \boldsymbol{\beta}, \boldsymbol{\mu}, \boldsymbol{\mu}_{0}\right) \boldsymbol{u}+\boldsymbol{q}_{2}^{\prime}\left(\boldsymbol{\alpha}, \boldsymbol{\beta}, \boldsymbol{\mu}, \boldsymbol{\mu}_{0}\right) \boldsymbol{v} \\
0=\beta_{1}(\boldsymbol{v}, \boldsymbol{\mu})-\boldsymbol{q}_{0}(\boldsymbol{v}, \boldsymbol{\mu}) .
\end{gathered}
$$

Let $\gamma(\boldsymbol{\eta}, \boldsymbol{\xi})=\gamma(\boldsymbol{x}), \beta_{\rho+1}(\boldsymbol{v}, \boldsymbol{\mu})=\boldsymbol{u}(\boldsymbol{v}, \boldsymbol{\mu})-$ $\gamma(\boldsymbol{\alpha}(\boldsymbol{v}, \boldsymbol{\mu}), \boldsymbol{\beta}(\boldsymbol{v}, \boldsymbol{\mu})) \boldsymbol{z}(\boldsymbol{v}, \boldsymbol{\mu})$ and

$$
\chi(\boldsymbol{v}, \boldsymbol{\mu})=\left(\begin{array}{c}
\chi_{1}(\boldsymbol{v}, \boldsymbol{\mu}) \\
\vdots \\
\chi_{\rho-1}(\boldsymbol{v}, \boldsymbol{\mu}) \\
\chi_{\rho}(\boldsymbol{v}, \boldsymbol{\mu})
\end{array}\right)=\left(\begin{array}{c}
\beta_{2}(\boldsymbol{v}, \boldsymbol{\mu}) \\
\vdots \\
\beta_{\rho}(\boldsymbol{v}, \boldsymbol{\mu}) \\
\beta_{\rho+1}(\boldsymbol{v}, \boldsymbol{\mu})
\end{array}\right) .
$$

Assumption 4 For all $\boldsymbol{v} \in V$, there exist a set of real numbers $a_{0}^{i}, a_{1}^{i}, \cdots, a_{\varrho_{i}-1}^{i}$ such that, for $i=1, \ldots, \rho$,

$$
L_{\boldsymbol{S} \boldsymbol{v}}^{\varrho_{i}} \chi_{i}=a_{0}^{i} \chi_{i}+a_{1}^{i} L_{\boldsymbol{S} \boldsymbol{v}} \chi_{i}+\cdots+a_{\varrho_{i}-1}^{i} L_{\boldsymbol{S} \boldsymbol{v}}^{\varrho_{i}-1} \chi_{i} .
$$

Remark 3 Assumption 3 is standard and necessary for the solvability of the robust output regulation problem of singular nonlinear systems [21,24], and the equations (18) are called singular regulator equations. Assumption 4 holds if the solution of the singular regulator equations in (18) is polynomial in $v$ which is used in the literature on the robust output regulation problem for normal nonlinear systems. This additional condition is also required to solve the robust output regulation problem for singular nonlinear systems $[21,24]$.
Define $\boldsymbol{\tau}_{i}(\boldsymbol{v}, \boldsymbol{\mu}) \in \Re^{\varrho_{i}}$ as

$$
\boldsymbol{\tau}_{i}(\boldsymbol{v}, \boldsymbol{\mu})=\left(\begin{array}{c}
\chi_{i}(\boldsymbol{v}, \boldsymbol{\mu}) \\
L_{\boldsymbol{S} \boldsymbol{v} \chi_{i}} \\
\vdots \\
L_{\boldsymbol{S} \boldsymbol{v}}^{\varrho_{i}-1} \chi_{i}
\end{array}\right), \quad i=1, \ldots, \rho
$$

it follows that

$$
\begin{gathered}
\frac{\partial \boldsymbol{\tau}_{i}(\boldsymbol{v}, \boldsymbol{\mu})}{\partial \boldsymbol{v}} \boldsymbol{S} \boldsymbol{v}=\boldsymbol{\Phi}_{i} \boldsymbol{\tau}_{i}(\boldsymbol{v}, \boldsymbol{\mu}) \\
\chi_{i}=\boldsymbol{\Psi}_{i} \boldsymbol{\tau}_{i}, i=1, \ldots, \rho
\end{gathered}
$$

where

$$
\begin{gathered}
\boldsymbol{\Phi}_{i}=\left(\begin{array}{ccccc}
0 & 1 & 0 & \ldots & 0 \\
0 & 0 & 1 & \ldots & 0 \\
\cdot & \cdot & \cdot & \ldots & \cdot \\
0 & 0 & 0 & \ldots & 1 \\
a_{0}^{i} & a_{1}^{i} & a_{2}^{i} & \ldots & a_{\varrho_{i}-1}^{i}
\end{array}\right) \\
\boldsymbol{\Psi}_{i}=\left(\begin{array}{lllll}
1 & 0 & 0 & \cdots & 0
\end{array}\right) .
\end{gathered}
$$

The Sylvester equation

$$
\boldsymbol{T}_{0}^{i} \boldsymbol{\Phi}_{i}-\boldsymbol{M}_{i} \boldsymbol{T}_{0}^{i}=\boldsymbol{N}_{i} \boldsymbol{\Psi}_{i}, \quad i=1, \ldots, \rho
$$

has a unique nonsingular solution $T_{0}^{i}$ for a given controllable pair $\left(\boldsymbol{M}_{i}, \boldsymbol{N}_{i}\right)$ where $\boldsymbol{M}_{i}$ is a Hurwitz matrix and $\boldsymbol{N}_{i}$ is a vector of appropriate dimension. Let $\boldsymbol{\theta}_{i}(\boldsymbol{v}, \boldsymbol{\mu})=$ $\boldsymbol{T}_{0}^{i} \boldsymbol{\tau}_{i}(\boldsymbol{v}, \boldsymbol{\mu})$ and $\xi_{\rho+1}=u-\gamma(\boldsymbol{\eta}, \boldsymbol{\xi}) z$, then we have

$$
\begin{gathered}
\chi_{i}=\boldsymbol{\Psi}_{i}\left(\boldsymbol{T}_{0}^{i}\right)^{-1} \boldsymbol{\theta}_{i}, \quad i=1, \ldots, \rho \\
\dot{\boldsymbol{\theta}}_{i}=\boldsymbol{M}_{i} \boldsymbol{\theta}_{i}+\boldsymbol{N}_{i} \boldsymbol{\Psi}_{i}\left(\boldsymbol{T}_{0}^{i}\right)^{-1} \boldsymbol{\theta}_{i}=\boldsymbol{M}_{i} \boldsymbol{\theta}_{i}+\boldsymbol{N}_{i} \chi_{i}
\end{gathered}
$$

and we can define the following dynamic as internal model:

$$
\dot{\boldsymbol{\sigma}}_{i}=\boldsymbol{M}_{i} \boldsymbol{\sigma}_{i}+\boldsymbol{N}_{i} \xi_{i+1}, \quad \boldsymbol{\sigma}_{i} \in \Re^{\varrho_{i}}, \quad i=1, \ldots, \rho .
$$

Attaching the internal model (19) to the system (17) and performing the following coordinate and input transformation

$$
\begin{gathered}
\overline{\boldsymbol{\sigma}}_{i}=\boldsymbol{\sigma}_{i}-\boldsymbol{\theta}_{i}(\boldsymbol{v}, \boldsymbol{\mu}) \\
\overline{\boldsymbol{\eta}}=\boldsymbol{\eta}-\boldsymbol{\alpha}(\boldsymbol{v}, \boldsymbol{\mu}) \\
\bar{\xi}_{1}=\xi_{1}-\beta_{1}(\boldsymbol{v}, \boldsymbol{\mu})=e \\
\bar{\xi}_{i+1}=\xi_{i+1}-\boldsymbol{\Psi}_{i}\left(\boldsymbol{T}_{0}^{i}\right)^{-1} \boldsymbol{\sigma}_{i}, \quad i=1, \ldots, \rho
\end{gathered}
$$

yield an augmented system as

$$
\begin{gathered}
\dot{\overline{\boldsymbol{\eta}}}=\overline{\boldsymbol{\psi}}\left(\overline{\boldsymbol{\eta}}, \overline{\boldsymbol{\xi}}, \overline{\boldsymbol{\sigma}}, \boldsymbol{v}, \boldsymbol{\mu}, \boldsymbol{\mu}_{0}\right) \\
\dot{\bar{\sigma}}_{i}=\left(\boldsymbol{M}_{i}+\boldsymbol{N}_{i} \boldsymbol{\Psi}_{i}\left(\boldsymbol{T}_{0}^{i}\right)^{-1}\right) \overline{\boldsymbol{\sigma}}_{i}+\boldsymbol{N}_{i} \overline{\bar{\xi}}_{i+1}, i=1, \ldots, \rho \\
\dot{\bar{\xi}}_{i}=\bar{\xi}_{i+1}+\bar{\varphi}_{i}\left(\overline{\boldsymbol{\eta}}, \overline{\boldsymbol{\xi}}, \overline{\boldsymbol{\sigma}}, \boldsymbol{v}, \boldsymbol{\mu}, \boldsymbol{\mu}_{0}\right), \quad i=1, \ldots, \rho-1 \\
\dot{\bar{\xi}}_{\rho}=\boldsymbol{f}_{3}\left(\boldsymbol{\eta}, \boldsymbol{\xi}, \boldsymbol{\mu}, \boldsymbol{\mu}_{0}\right)+\boldsymbol{p}_{3}\left(\boldsymbol{\eta}, \boldsymbol{\xi}, \boldsymbol{\mu}, \boldsymbol{\mu}_{0}\right) \boldsymbol{z}+
\end{gathered}
$$




$$
\begin{gathered}
\boldsymbol{g}_{3}\left(\boldsymbol{\eta}, \boldsymbol{\xi}, \boldsymbol{\mu}, \boldsymbol{\mu}_{0}\right)\left(\bar{\xi}_{\rho+1}+\boldsymbol{\Psi}_{\rho}\left(\boldsymbol{T}_{0}^{\rho}\right)^{-1} \boldsymbol{\sigma}_{\rho}+\gamma(\boldsymbol{\eta}, \boldsymbol{\xi}) \boldsymbol{z}\right)+ \\
\boldsymbol{q}_{3}\left(\boldsymbol{\eta}, \boldsymbol{\xi}, \boldsymbol{\mu}, \boldsymbol{\mu}_{0}\right) \boldsymbol{v}-\boldsymbol{\Psi}_{\rho-1}\left(\boldsymbol{T}_{0}^{\rho-1}\right)^{-1} \dot{\boldsymbol{\sigma}}_{\rho-1} \\
\mathbf{0}=\boldsymbol{f}_{2}^{\prime}\left(\boldsymbol{\eta}, \boldsymbol{\xi}, \boldsymbol{\mu}, \boldsymbol{\mu}_{0}\right)+\boldsymbol{p}_{2}^{\prime}\left(\boldsymbol{\eta}, \boldsymbol{\xi}, \boldsymbol{\mu}, \boldsymbol{\mu}_{0}\right) \boldsymbol{z}+ \\
\boldsymbol{g}_{2}^{\prime}\left(\boldsymbol{\eta}, \boldsymbol{\xi}, \boldsymbol{\mu}, \boldsymbol{\mu}_{0}\right)\left(\bar{\xi}_{\rho+1}+\boldsymbol{\Psi}_{\rho}\left(\boldsymbol{T}_{0}^{\rho}\right)^{-1} \sigma_{\boldsymbol{\rho}}+\gamma(\boldsymbol{\eta}, \boldsymbol{\xi}) \boldsymbol{z}\right)+ \\
\boldsymbol{q}_{2}^{\prime}\left(\boldsymbol{\eta}, \boldsymbol{\xi}, \boldsymbol{\mu}, \boldsymbol{\mu}_{0}\right) \boldsymbol{v}
\end{gathered}
$$

where $\overline{\boldsymbol{\sigma}}=\operatorname{col}\left(\bar{\sigma}_{1}, \ldots, \bar{\sigma}_{\rho}\right), \overline{\boldsymbol{\xi}}=\operatorname{col}\left(\bar{\xi}_{1}, \ldots, \bar{\xi}_{\rho}\right)$, and

$$
\begin{gathered}
\overline{\boldsymbol{\psi}}\left(\overline{\boldsymbol{\eta}}, \overline{\boldsymbol{\xi}}, \boldsymbol{v}, \boldsymbol{\mu}, \boldsymbol{\mu}_{0}\right)= \\
\boldsymbol{\psi}\left(\overline{\boldsymbol{\eta}}+\boldsymbol{\alpha}(\boldsymbol{v}), \bar{\xi}_{1}+\beta_{1}(\boldsymbol{v}), \bar{\xi}_{2}+\boldsymbol{\Psi}_{1}\left(\boldsymbol{T}_{0}^{1}\right)^{-1} \boldsymbol{\sigma}_{1}, \ldots,\right. \\
\left.\bar{\xi}_{\rho}+\boldsymbol{\Psi}_{\rho-1}\left(\boldsymbol{T}_{0}^{\rho-1}\right)^{-1} \boldsymbol{\sigma}_{\rho-1}, \boldsymbol{v}, \boldsymbol{\mu}, \boldsymbol{\mu}_{0}\right)- \\
\boldsymbol{\psi}\left(\boldsymbol{\alpha}(\boldsymbol{v}), \beta_{1}(\boldsymbol{v}), \beta_{2}(\boldsymbol{v}), \ldots, \beta_{\rho}(\boldsymbol{v}), \boldsymbol{v}, \boldsymbol{\mu}, \boldsymbol{\mu}_{0}\right), \\
\bar{\varphi}_{1}\left(\overline{\boldsymbol{\eta}}, \overline{\boldsymbol{\xi}}, \overline{\boldsymbol{\sigma}}, \boldsymbol{v}, \boldsymbol{\mu}, \boldsymbol{\mu}_{0}\right)= \\
\varphi_{1}\left(\overline{\boldsymbol{\eta}}+\boldsymbol{\alpha}(\boldsymbol{v}), \bar{\xi}_{1}+\beta_{1}(\boldsymbol{v}), \bar{\xi}_{2}+\boldsymbol{\Psi}_{1}\left(\boldsymbol{T}_{0}^{1}\right)^{-1} \boldsymbol{\sigma}_{1}, \ldots,\right. \\
\left.\bar{\xi}_{\rho}+\boldsymbol{\Psi}_{\rho-1}\left(\boldsymbol{T}_{0}^{\rho-1}\right)^{-1} \boldsymbol{\sigma}_{\rho-1}, \boldsymbol{v}, \boldsymbol{\mu}, \boldsymbol{\mu}_{0}\right)+ \\
\boldsymbol{\Psi}_{1}\left(\boldsymbol{T}_{0}^{1}\right)^{-1} \boldsymbol{\sigma}_{1}-\frac{\partial \beta_{1}(\boldsymbol{v}, \boldsymbol{\mu})}{\partial \boldsymbol{v}} \boldsymbol{S} \boldsymbol{v}, \\
\bar{\varphi}_{i}\left(\overline{\boldsymbol{\eta}}, \overline{\boldsymbol{\xi}}, \overline{\boldsymbol{\sigma}}, \boldsymbol{v}, \boldsymbol{\mu}, \boldsymbol{\mu}_{0}\right)= \\
\varphi_{i}\left(\overline{\boldsymbol{\eta}}+\boldsymbol{\alpha}(\boldsymbol{v}), \bar{\xi}_{1}+\beta_{1}(\boldsymbol{v}), \bar{\xi}_{2}+\boldsymbol{\Psi}{ }_{1}\left(\boldsymbol{T}_{0}^{1}\right)^{-1} \boldsymbol{\sigma}_{1}, \ldots,\right. \\
\left.\bar{\xi}_{\rho}+\boldsymbol{\Psi}_{\rho-1}\left(\boldsymbol{T}_{0}^{\rho-1}\right)^{-1} \boldsymbol{\sigma}_{\rho-1}, \boldsymbol{v}, \boldsymbol{\mu}, \boldsymbol{\mu}_{0}\right)+ \\
\boldsymbol{\Psi}_{i}\left(\boldsymbol{T}_{0}^{i}\right)^{-1} \boldsymbol{\sigma}_{i}-\boldsymbol{\Psi}_{i-1}\left(\boldsymbol{T}_{0}^{i-1}\right)^{-1} \dot{\boldsymbol{\sigma}}_{i-1}, \\
i=2, \ldots, \rho-1 .
\end{gathered}
$$

It can be verified that $\operatorname{col}(\overline{\boldsymbol{\eta}}, \overline{\boldsymbol{\sigma}}, \overline{\boldsymbol{\xi}})=0$ is an equilibrium point of the system (21) with $\bar{\xi}_{\rho+1}=0$ for all $\boldsymbol{v} \in V$ and $\boldsymbol{\mu} \in U$. For the system (21), we can define a robust stabilization problem as follows.

Global robust stabilization problem Given the system (21), find a state feedback control law

$$
\bar{\xi}_{\rho+1}=\bar{k}\left(\bar{\xi}_{1}, \bar{\xi}_{2}, \ldots, \bar{\xi}_{\rho}\right)
$$

with $\bar{k}(0,0, \ldots, 0)=0$, such that for any initial conditions $\left(\overline{\boldsymbol{\eta}}(0), \overline{\boldsymbol{\sigma}}_{i}(0), \overline{\boldsymbol{\xi}}(0) \in \Re^{n-\rho} \times \Re^{\varrho_{i}} \times \Re^{\rho}\right.$, the equilibrium $\operatorname{col}(\overline{\boldsymbol{\eta}}, \overline{\boldsymbol{\sigma}}, \overline{\boldsymbol{\xi}})=0$ of the closed-loop system composed of (21) and (22) is global asymptotically stable for all $\boldsymbol{v} \in V$ and $\boldsymbol{\mu} \in U$.

Consider the following associate control law

$$
\begin{gathered}
\boldsymbol{T}\left(\boldsymbol{x}, \boldsymbol{\mu}_{0}\right)=\operatorname{col}(\boldsymbol{\eta}, \boldsymbol{\xi}) \\
\dot{\boldsymbol{\sigma}}_{i}=\boldsymbol{M}_{i} \boldsymbol{\sigma}_{i}+\boldsymbol{N}_{i} \xi_{i+1}, \quad i=1, \ldots, \rho \\
\xi_{\rho+1}=\bar{k}\left(e, \xi_{2}-\boldsymbol{\Psi}_{1}\left(\boldsymbol{T}_{0}^{1}\right)^{-1} \boldsymbol{\sigma}_{1}, \ldots,\right. \\
\left.\xi_{\rho}-\boldsymbol{\Psi}_{\rho-1}\left(\boldsymbol{T}_{0}^{\rho-1}\right)^{-1} \boldsymbol{\sigma}_{\rho-1}\right)+\boldsymbol{\Psi}_{\rho}\left(\boldsymbol{T}_{0}^{\rho}\right)^{-1} \boldsymbol{\sigma}_{\rho} \\
u=\xi_{\rho+1}+\boldsymbol{\gamma}(\boldsymbol{\eta}, \boldsymbol{\xi}) \boldsymbol{z}
\end{gathered}
$$

then we have the following result.

Theorem 1 Under Assumptions 1-4, if the global robust stabilization problem for the system (21) is solvable by the control law (22), then the global robust output regulation problem for systems (1) and (2) is solvable by the control law (23).

Proof For all $\boldsymbol{v} \in V$ and $\boldsymbol{\mu} \in U, \boldsymbol{\alpha}(\boldsymbol{v}, \boldsymbol{\mu}), \boldsymbol{\beta}(\boldsymbol{v}, \boldsymbol{\mu})$ and $\boldsymbol{\theta}_{i}(\boldsymbol{v}, \boldsymbol{\mu})$ are bounded. If for any initial conditions $\left(\overline{\boldsymbol{\eta}}(0), \overline{\boldsymbol{\sigma}}_{i}(0), \overline{\boldsymbol{\xi}}(0)\right) \in \Re^{n-\rho} \times \Re^{\varrho_{i}} \times \Re^{\rho}$, the equilibrium $\operatorname{col}(\overline{\boldsymbol{\eta}}, \overline{\boldsymbol{\sigma}}, \overline{\boldsymbol{\xi}})=0$ of the closed-loop system composed of (21) and (22) is global asymptotically stable for all $v \in V$ and $\boldsymbol{\mu} \in U$, then by the transformation (20), there exists unique and bounded $\left(\boldsymbol{\eta}, \xi_{1}, \ldots, \xi_{\rho}\right)$ for the closed-loop system composed of systems (17) and (23). By the global diffeomorphism $\boldsymbol{T}\left(\boldsymbol{x}, \boldsymbol{\mu}_{0}\right)$, for any initial state $x(0) \in \Re^{n}$, $z(0) \in \Re^{s}, \sigma_{i}(0) \in \Re^{\varrho_{i}}$, and for all $\boldsymbol{v}(t) \in V$ and $\boldsymbol{\mu} \in$ $U$, the trajectories of the closed-loop systems composed of (1), (2) and (23) are unique and bounded. Moreover, $\lim _{t \rightarrow \infty} e(t)=\lim _{t \rightarrow \infty}(h(\boldsymbol{x}(t))-q(\boldsymbol{v}(t), \boldsymbol{\mu}))=\lim _{t \rightarrow \infty} \bar{\xi}_{1}(t)=0$.

\section{Solvability conditions}

In previous section, the global robust output regulation problem of a singular nonlinear system is converted into a global robust stabilization problem of an augmented singular nonlinear system, that is, to establish the solvability conditions of the global robust output regulation problem of the system (1), it is sufficient to address the solution of the global robust stabilization problem of the system (21). Under Assumption 1, it can be verified that, for system (21),

$$
\begin{gathered}
\boldsymbol{z}=\left(\boldsymbol{p}_{2}^{\prime}\left(\boldsymbol{\eta}, \boldsymbol{\xi}, \boldsymbol{\mu}, \boldsymbol{\mu}_{0}\right)+\boldsymbol{g}_{2}^{\prime}\left(\boldsymbol{\eta}, \boldsymbol{\xi}, \boldsymbol{\mu}, \boldsymbol{\mu}_{0}\right) \boldsymbol{\gamma}(\boldsymbol{\eta}, \boldsymbol{\xi})\right)^{-1} . \\
\left(\boldsymbol{f}_{2}^{\prime}\left(\boldsymbol{\eta}, \boldsymbol{\xi}, \boldsymbol{\mu}, \boldsymbol{\mu}_{0}\right)+\boldsymbol{q}_{2}^{\prime}\left(\boldsymbol{\eta}, \boldsymbol{\xi}, \boldsymbol{\mu}, \boldsymbol{\mu}_{0}\right) \boldsymbol{v}+\right. \\
\left.\boldsymbol{g}_{2}^{\prime}\left(\boldsymbol{\eta}, \boldsymbol{\xi}, \boldsymbol{\mu}, \boldsymbol{\mu}_{0}\right)\left(\bar{\xi}_{\rho+1}+\boldsymbol{\Psi}_{\rho}\left(\boldsymbol{T}_{0}^{\rho}\right)^{-1} \boldsymbol{\sigma}_{\rho}\right)\right)
\end{gathered}
$$

hence, the system (21) can be written as

$$
\begin{gathered}
\dot{\overline{\boldsymbol{\eta}}}=\overline{\boldsymbol{\psi}}\left(\overline{\boldsymbol{\eta}}, \overline{\boldsymbol{\xi}}, \overline{\boldsymbol{\sigma}}, \boldsymbol{v}, \boldsymbol{\mu}, \boldsymbol{\mu}_{0}\right) \\
\dot{\overline{\boldsymbol{\sigma}}}_{i}=\left(\boldsymbol{M}_{i}+\boldsymbol{N}_{i} \boldsymbol{\Psi}_{i}\left(\boldsymbol{T}_{0}^{i}\right)^{-1}\right) \overline{\boldsymbol{\sigma}}_{i}+N_{i} \bar{\xi}_{i+1}, \quad i=1, \ldots, \rho \\
\dot{\bar{\xi}}_{i}=\bar{\xi}_{i+1}+\bar{\varphi}_{i}\left(\overline{\boldsymbol{\eta}}, \overline{\boldsymbol{\xi}}, \overline{\boldsymbol{\sigma}}, \boldsymbol{v}, \boldsymbol{\mu}, \boldsymbol{\mu}_{0}\right), \quad i=1, \ldots, \rho-1 \\
\dot{\bar{\xi}}_{\rho}=\bar{b}\left(\overline{\boldsymbol{\eta}}, \overline{\boldsymbol{\xi}}, \overline{\boldsymbol{\sigma}}_{1}, \ldots, \overline{\boldsymbol{\sigma}}_{\rho}, \boldsymbol{v}, \boldsymbol{\mu}, \boldsymbol{\mu}_{0}\right) \bar{\xi}_{\rho+1}+ \\
\bar{\varphi}_{\rho}\left(\overline{\boldsymbol{\eta}}, \overline{\boldsymbol{\xi}}, \overline{\boldsymbol{\sigma}}_{1}, \ldots, \overline{\boldsymbol{\sigma}}_{\rho}, \boldsymbol{v}, \boldsymbol{\mu}, \boldsymbol{\mu}_{0}\right)
\end{gathered}
$$

where

$$
\begin{gathered}
\bar{b}\left(\overline{\boldsymbol{\eta}}, \overline{\boldsymbol{\xi}}, \overline{\boldsymbol{\sigma}}_{1}, \ldots, \overline{\boldsymbol{\sigma}}_{\rho}, \boldsymbol{v}, \boldsymbol{\mu}, \boldsymbol{\mu}_{0}\right)= \\
\boldsymbol{g}_{3}\left(\boldsymbol{\eta}, \boldsymbol{\xi}, \boldsymbol{\mu}, \boldsymbol{\mu}_{0}\right)+\boldsymbol{\kappa}\left(\boldsymbol{\eta}, \boldsymbol{\xi}, \boldsymbol{\mu}, \boldsymbol{\mu}_{0}\right) \boldsymbol{g}_{2}^{\prime}\left(\boldsymbol{\eta}, \boldsymbol{\xi}, \boldsymbol{\mu}, \boldsymbol{\mu}_{0}\right) \\
\bar{\varphi} \bar{\rho}_{\rho}\left(\overline{\boldsymbol{\eta}}, \overline{\boldsymbol{\xi}}, \overline{\boldsymbol{\sigma}}_{1}, \ldots, \overline{\boldsymbol{\sigma}}_{\rho}, \boldsymbol{v}, \boldsymbol{\mu}, \boldsymbol{\mu}_{0}\right)= \\
\boldsymbol{f}_{3}\left(\boldsymbol{\eta}, \boldsymbol{\xi}, \boldsymbol{\mu}, \boldsymbol{\mu}_{0}\right)+\boldsymbol{g}_{3}\left(\boldsymbol{\eta}, \boldsymbol{\xi}, \boldsymbol{\mu}, \boldsymbol{\mu}_{0}\right) \boldsymbol{\Psi}_{\rho}\left(\boldsymbol{T}_{0}^{\rho}\right)^{-1} \boldsymbol{\sigma}_{\rho}+ \\
\boldsymbol{q}_{3}\left(\boldsymbol{\eta}, \boldsymbol{\xi}, \boldsymbol{\mu}, \boldsymbol{\mu}_{0}\right) \boldsymbol{v}+
\end{gathered}
$$




$$
\begin{gathered}
\kappa\left(\boldsymbol{\eta}, \boldsymbol{\xi}, \boldsymbol{\mu}, \boldsymbol{\mu}_{0}\right)\left(\boldsymbol{f}_{2}^{\prime}\left(\boldsymbol{\eta}, \boldsymbol{\xi}, \boldsymbol{\mu}, \boldsymbol{\mu}_{0}\right)+\boldsymbol{q}_{2}^{\prime}\left(\boldsymbol{\eta}, \boldsymbol{\xi}, \boldsymbol{\mu}, \boldsymbol{\mu}_{0}\right) \boldsymbol{v}\right)+ \\
\boldsymbol{\kappa}\left(\boldsymbol{\eta}, \boldsymbol{\xi}, \boldsymbol{\mu}, \boldsymbol{\mu}_{0}\right) \boldsymbol{g}_{2}^{\prime}\left(\boldsymbol{\eta}, \boldsymbol{\xi}, \boldsymbol{\mu}, \boldsymbol{\mu}_{0}\right) \boldsymbol{\Psi}_{\rho}\left(\boldsymbol{T}_{0}^{\rho}\right)^{-1} \boldsymbol{\sigma}_{\rho}- \\
\boldsymbol{\Psi}_{\rho-1}\left(\boldsymbol{T}_{0}^{\rho-1}\right)^{-1} \dot{\boldsymbol{\sigma}}_{\rho-1}
\end{gathered}
$$

with

$$
\begin{gathered}
\boldsymbol{\kappa}\left(\boldsymbol{\eta}, \boldsymbol{\xi}, \boldsymbol{\mu}, \boldsymbol{\mu}_{0}\right)= \\
\left(\boldsymbol{p}_{3}\left(\boldsymbol{\eta}, \boldsymbol{\xi}, \boldsymbol{\mu}, \boldsymbol{\mu}_{0}\right)+\boldsymbol{g}_{3}\left(\boldsymbol{\eta}, \boldsymbol{\xi}, \boldsymbol{\mu}, \boldsymbol{\mu}_{0}\right) \gamma(\boldsymbol{\eta}, \boldsymbol{\xi})\right) . \\
\left(\boldsymbol{p}_{2}^{\prime}\left(\boldsymbol{\eta}, \boldsymbol{\xi}, \boldsymbol{\mu}, \boldsymbol{\mu}_{0}\right)+\boldsymbol{g}_{2}^{\prime}\left(\boldsymbol{\eta}, \boldsymbol{\xi}, \boldsymbol{\mu}, \boldsymbol{\mu}_{0}\right) \boldsymbol{\gamma}^{-1}(\boldsymbol{\eta}, \boldsymbol{\xi})\right) .
\end{gathered}
$$

Thus, the global robust stabilization problem of (21) is equivalent to the global stabilization problem of (24). To solve the global robust stabilization problem of system (24), we further need some assumptions as follows.

Assumption 5 For the system (24), there exist $\frac{\partial \bar{\psi}}{\partial \bar{\xi}_{k+1}}=0$ and $\frac{\partial \bar{\varphi}_{k}}{\partial \bar{\xi}_{j}}=0$ with $k=1, \ldots, \rho-1, j=$ $k+1, \ldots, \rho$.

Assumption 6 For all $\boldsymbol{v} \in V$ and $\boldsymbol{\mu} \in U$, the system $\dot{\overline{\boldsymbol{\eta}}}=\overline{\boldsymbol{\psi}}\left(\overline{\boldsymbol{\eta}}, \bar{\xi}_{1}, \boldsymbol{v}, \boldsymbol{\mu}, \boldsymbol{\mu}_{0}\right)$ is robustly input-to-state stable with $\bar{\eta}$ as state and $\bar{\xi}_{1}$ as input, and has a known continuously differential $\left(C^{1}\right)$ class $\mathcal{K}_{\infty}$ gain function $\kappa_{0}(\cdot)$.

Assumption 7 For all $\boldsymbol{v} \in V$ and $\boldsymbol{\mu} \in U$, there exist locally Lipschitz class $\mathcal{K}$ functions $\rho_{0}(\cdot)$ and $\rho_{1}(\cdot)$ such that

$$
\begin{gathered}
\max \left\{\left|\bar{\varphi}_{\rho}\left(\overline{\boldsymbol{\eta}}, \overline{\boldsymbol{\xi}}, \overline{\boldsymbol{\sigma}}_{1}, \ldots, \overline{\boldsymbol{\sigma}}_{\rho}, \boldsymbol{v}, \boldsymbol{\mu}, \boldsymbol{\mu}_{0}\right)\right|,\right. \\
\left.\left|\bar{\xi}_{\rho}\right|\left|\bar{b}\left(\overline{\boldsymbol{\eta}}, \overline{\boldsymbol{\xi}}_{,}, \overline{\boldsymbol{\sigma}}_{1}, \ldots, \overline{\boldsymbol{\sigma}}_{\rho}, \boldsymbol{v}, \boldsymbol{\mu}, \boldsymbol{\mu}_{0}\right)\right|^{2}\right\} \leqslant \\
\max \left\{\rho_{0}\left(\left|\bar{\xi}_{\rho}\right|\right), \rho_{1}\left(\left|\operatorname{col}\left(\overline{\boldsymbol{\eta}}, \bar{\xi}_{1}, \ldots, \bar{\xi}_{\rho-1}, \overline{\boldsymbol{\sigma}}\right)\right|\right)\right\} .
\end{gathered}
$$

Assumption 8 For all $\boldsymbol{v} \in V$ and $\boldsymbol{\mu} \in U$, $\bar{b}\left(\overline{\boldsymbol{\eta}}, \overline{\boldsymbol{\xi}}, \overline{\boldsymbol{\sigma}}_{1}, \ldots, \overline{\boldsymbol{\sigma}}_{\rho}, \boldsymbol{v}, \boldsymbol{\mu}, \boldsymbol{\mu}_{0}\right)>0$.

Remark 4 The above assumptions are required to establish the solvablility conditions for the robust stabilization problems of the augmented system (24). The assumptions are made with respect to the augmented system but not the original system because we need to know the structure of the system such that we can establish the solvability conditions of the robust stabilization problem. Under Assumption 5, it can be verified that the $\bar{\eta}$ and $\bar{\xi}_{i}$ subsystem of the system (24) can be written as $\dot{\overline{\boldsymbol{\eta}}}=\overline{\boldsymbol{\psi}}\left(\overline{\boldsymbol{\eta}}, \bar{\xi}_{1}, \boldsymbol{v}, \boldsymbol{\mu}, \boldsymbol{\mu}_{0}\right)$, and $\dot{\bar{\xi}}_{i}=\bar{\xi}_{i+1}+\bar{\varphi}_{i}\left(\overline{\boldsymbol{\eta}}, \bar{\xi}_{1}, \ldots, \bar{\xi}_{i}, \overline{\boldsymbol{\sigma}}_{1}, \ldots, \overline{\boldsymbol{\sigma}}_{i}, \boldsymbol{v}, \boldsymbol{\mu}, \boldsymbol{\mu}_{0}\right)$, hence the flow of the state $\bar{\eta}$ is only affected by $\bar{\xi}_{1}$. Assumptions $6-8$ are quite familiar in the literature on semiglobal or global stabilization of nonlinear systems.

Theorem 2 Under Assumptions 5-8, the global robust stabilization problem of the system (21) is solvable by the control law (22).

Proof Since the global robust stabilization problem of (21) is equivalent to the global stabilization problem of (24), we will focus on the solution of the global robust stabilization problem for (24). To this end, let us perform the following coordinate transformation on (24) which can be done because of Assumption 5,

$$
\begin{gathered}
\tilde{\boldsymbol{\sigma}}_{i}=\overline{\boldsymbol{\sigma}}_{i}-\boldsymbol{N}_{i} \bar{\xi}_{i}, \quad i=1, \ldots, \rho-1 \\
B\left(\overline{\boldsymbol{\eta}}, \overline{\boldsymbol{\xi}}, \overline{\boldsymbol{\sigma}}, \boldsymbol{v}, \boldsymbol{\mu}, \boldsymbol{\mu}_{0}\right)= \\
\int_{0}^{\bar{\xi}_{\rho}} \frac{1}{\bar{b}\left(\overline{\boldsymbol{\eta}}_{,} \bar{\xi}_{1}, \ldots, \bar{\xi}_{\rho-1}, s, \overline{\boldsymbol{\sigma}}_{1}, \ldots, \overline{\boldsymbol{\sigma}}_{\rho}, \boldsymbol{v}, \boldsymbol{\mu}, \boldsymbol{\mu}_{0}\right)} \mathrm{d} s \\
\tilde{\boldsymbol{\sigma}}_{\rho}=\overline{\boldsymbol{\sigma}}_{\rho}-\boldsymbol{N}_{\rho} B\left(\overline{\boldsymbol{\eta}}, \overline{\boldsymbol{\xi}}, \overline{\boldsymbol{\sigma}}, \boldsymbol{v}, \boldsymbol{\mu}, \boldsymbol{\mu}_{0}\right) .
\end{gathered}
$$

The coordinate transformation yields

$$
\begin{gathered}
\dot{\overline{\boldsymbol{\eta}}}=\overline{\boldsymbol{\psi}}\left(\overline{\boldsymbol{\eta}}, \bar{\xi}_{1}, \boldsymbol{v}, \boldsymbol{\mu}, \boldsymbol{\mu}_{0}\right) \\
\dot{\tilde{\boldsymbol{\sigma}}}_{i}=\boldsymbol{Q}_{i}\left(\overline{\boldsymbol{\eta}}, \bar{\xi}_{1}, \ldots, \bar{\xi}_{i}, \tilde{\boldsymbol{\sigma}}_{1}, \ldots, \tilde{\boldsymbol{\sigma}}_{i}, \boldsymbol{v}, \boldsymbol{\mu}, \boldsymbol{\mu}_{0}\right) \\
\dot{\bar{\xi}}_{i}=\bar{\xi}_{i+1}+\bar{\varphi}_{i}^{\circ}\left(\overline{\boldsymbol{\eta}}, \bar{\xi}_{1}, \ldots, \bar{\xi}_{i}, \tilde{\boldsymbol{\sigma}}_{1}, \ldots, \tilde{\boldsymbol{\sigma}}_{i}, \boldsymbol{v}, \boldsymbol{\mu}, \boldsymbol{\mu}_{0}\right) \\
\dot{\tilde{\boldsymbol{\sigma}}}_{\rho}=Q_{\rho}\left(\overline{\boldsymbol{\eta}}, \bar{\xi}_{1}, \ldots, \bar{\xi}_{\rho}, \tilde{\boldsymbol{\sigma}}, \boldsymbol{v}, \boldsymbol{\mu}, \boldsymbol{\mu}_{0}\right) \\
\dot{\bar{\xi}}_{\rho}=\bar{b}^{\circ}\left(\overline{\boldsymbol{\eta}}, \bar{\xi}_{1}, \ldots, \bar{\xi}_{\rho}, \tilde{\boldsymbol{\sigma}}, \boldsymbol{v}, \boldsymbol{\mu}, \boldsymbol{\mu}_{0}\right) \bar{\xi}_{\rho+1}+ \\
\bar{\varphi}_{\rho}^{\circ}\left(\overline{\boldsymbol{\eta}}, \bar{\xi}_{1}, \ldots, \bar{\xi}_{\rho}, \tilde{\boldsymbol{\sigma}}, \boldsymbol{v}, \boldsymbol{\mu}, \boldsymbol{\mu}_{0}\right)
\end{gathered}
$$

where $i=1, \ldots, \rho-1, \tilde{\boldsymbol{\sigma}}=\operatorname{col}\left(\tilde{\boldsymbol{\sigma}}_{1}, \ldots, \tilde{\boldsymbol{\sigma}}_{\rho}\right)$ and

$$
\begin{gathered}
\boldsymbol{Q}_{i}\left(\overline{\boldsymbol{\eta}}, \bar{\xi}_{1}, \ldots, \bar{\xi}_{i}, \tilde{\boldsymbol{\sigma}}_{1}, \ldots, \tilde{\boldsymbol{\sigma}}_{i}, \boldsymbol{v}, \boldsymbol{\mu}, \boldsymbol{\mu}_{0}\right)= \\
\left(\boldsymbol{M}_{i}+\boldsymbol{N}_{i} \boldsymbol{\Psi}_{i}\left(\boldsymbol{T}_{0}^{i}\right)^{-1}\right) \overline{\boldsymbol{\sigma}}_{i}- \\
\boldsymbol{N}_{i} \bar{\varphi}_{i}\left(\overline{\boldsymbol{\eta}}, \bar{\xi}_{1}, \ldots, \bar{\xi}_{i}, \overline{\boldsymbol{\sigma}}_{1}, \ldots, \overline{\boldsymbol{\sigma}}_{i}, \boldsymbol{v}, \boldsymbol{\mu}, \boldsymbol{\mu}_{0}\right), \\
\bar{\varphi}_{i}^{\circ}\left(\overline{\boldsymbol{\eta}}, \bar{\xi}_{1}, \ldots, \bar{\xi}_{i}, \tilde{\boldsymbol{\sigma}}_{1}, \ldots, \tilde{\boldsymbol{\sigma}}_{i}, \boldsymbol{v}, \boldsymbol{\mu}, \boldsymbol{\mu}_{0}\right)= \\
\bar{\varphi}_{i}\left(\overline{\boldsymbol{\eta}}_{,} \bar{\xi}_{1}, \ldots, \bar{\xi}_{i}, \tilde{\boldsymbol{\sigma}}_{1}+N_{1} \bar{\xi}_{1}, \ldots, \tilde{\boldsymbol{\sigma}}_{i}+N_{i} \bar{\xi}_{i}, \boldsymbol{v}, \boldsymbol{\mu}, \boldsymbol{\mu}_{0}\right), \\
\boldsymbol{Q}_{\rho}\left(\overline{\boldsymbol{\eta}}, \bar{\xi}_{1}, \ldots, \bar{\xi}_{\rho}, \tilde{\boldsymbol{\sigma}}, \boldsymbol{v}, \boldsymbol{\mu}, \boldsymbol{\mu}_{0}\right)= \\
\left(\boldsymbol{M}_{\rho}+\boldsymbol{N}_{\rho} \boldsymbol{\Psi}_{\rho}\left(\boldsymbol{T}_{0}^{\rho}\right)^{-1}\right) \overline{\boldsymbol{\sigma}}_{\rho}- \\
\boldsymbol{N}_{\rho}\left\{\bar{b}\left(\overline{\boldsymbol{\eta}}, \overline{\boldsymbol{\xi}}, \overline{\boldsymbol{\sigma}}_{1}, \ldots, \overline{\boldsymbol{\sigma}}_{\rho}, \boldsymbol{v}, \boldsymbol{\mu}, \boldsymbol{\mu}_{0}\right)\right\} \\
\bar{\varphi} \rho\left(\overline{\boldsymbol{\eta}}, \overline{\boldsymbol{\xi}}, \overline{\boldsymbol{\sigma}}_{1}, \ldots, \overline{\boldsymbol{\sigma}}_{\rho}, \boldsymbol{v}, \boldsymbol{\mu}, \boldsymbol{\mu}_{0}\right) \\
\bar{b}^{\circ}\left(\overline{\boldsymbol{\eta}}, \bar{\xi}_{1}, \ldots, \bar{\xi}_{\rho}, \tilde{\boldsymbol{\sigma}}, \boldsymbol{v}, \boldsymbol{\mu}, \boldsymbol{\mu}_{0}\right)= \\
\bar{b}\left(\overline{\boldsymbol{\eta}}, \overline{\boldsymbol{\xi}}, \tilde{\boldsymbol{\sigma}}_{1}+\boldsymbol{N}_{1} \bar{\xi}_{1}, \ldots,\right. \\
\left.\tilde{\boldsymbol{\sigma}}_{\rho}+\boldsymbol{N}_{\rho} B\left(\overline{\boldsymbol{\eta}}, \overline{\boldsymbol{\xi}}, \overline{\boldsymbol{\sigma}}, \boldsymbol{v}, \boldsymbol{\mu}^{*}\right), \boldsymbol{v}, \boldsymbol{\mu}, \boldsymbol{\mu}_{0}\right), \\
\bar{\varphi}_{\rho}^{\circ}\left(\overline{\boldsymbol{\eta}}, \bar{\xi}_{1}, \ldots, \bar{\xi}_{\rho}, \tilde{\boldsymbol{\sigma}}, \boldsymbol{v}, \boldsymbol{\mu}, \boldsymbol{\mu}_{0}\right)= \\
\bar{\varphi}_{\rho}\left(\overline{\boldsymbol{\eta}}, \overline{\boldsymbol{\xi}}, \tilde{\boldsymbol{\sigma}}_{1}+\boldsymbol{N}_{1} \bar{\xi}_{1}, \ldots,\right. \\
\left.\tilde{\boldsymbol{\sigma}}_{\rho}+\boldsymbol{N}_{\rho} B\left(\overline{\boldsymbol{\eta}}, \overline{\boldsymbol{\xi}}, \overline{\boldsymbol{\sigma}}, \boldsymbol{v}, \boldsymbol{\mu}^{*}\right), \boldsymbol{v}, \boldsymbol{\mu}, \boldsymbol{\mu}_{0}\right) .
\end{gathered}
$$

Consider another transformation

$$
\begin{gathered}
\tilde{\xi}_{1}=\bar{\xi}_{1}, \\
\tilde{\xi}_{j}=\bar{\xi}_{j}-\alpha_{j-1}\left(\tilde{\xi}_{j-1}\right), j=2, \ldots, \rho
\end{gathered}
$$

where $\alpha_{j-1}\left(\tilde{\xi}_{j-1}\right)=-\tilde{\xi}_{j-1} \rho_{j-1}\left(\tilde{\xi}_{j-1}\right)$ and $\rho_{j-1}\left(\tilde{\xi}_{j-1}\right) \geqslant 0$ are some smooth scalar functions, it follows that, for $i=1, \ldots, \rho-1$,

$$
\dot{\overline{\boldsymbol{\eta}}}=\overline{\boldsymbol{\psi}}\left(\overline{\boldsymbol{\eta}}, \tilde{\xi}_{1}, \boldsymbol{v}, \boldsymbol{\mu}, \boldsymbol{\mu}_{0}\right)
$$




$$
\begin{aligned}
& \dot{\tilde{\boldsymbol{\sigma}}}_{i}=\tilde{\boldsymbol{Q}}_{i}\left(\overline{\boldsymbol{\eta}}, \tilde{\xi}_{1}, \ldots, \tilde{\xi}_{i}, \tilde{\boldsymbol{\sigma}}_{1}, \ldots, \tilde{\boldsymbol{\sigma}}_{i}, \boldsymbol{v}, \boldsymbol{\mu}, \boldsymbol{\mu}_{0}\right) \\
& \dot{\tilde{\xi}}_{i}=\tilde{\xi}_{i+1}+\tilde{\varphi}_{i}\left(\overline{\boldsymbol{\eta}}, \tilde{\xi}_{1}, \ldots, \tilde{\xi}_{i}, \tilde{\boldsymbol{\sigma}}_{1}, \ldots, \tilde{\boldsymbol{\sigma}}_{i}, \boldsymbol{v}, \boldsymbol{\mu}, \boldsymbol{\mu}_{0}\right) \\
& \dot{\tilde{\boldsymbol{\sigma}}}_{\rho}=\tilde{\boldsymbol{Q}}_{\rho}\left(\overline{\boldsymbol{\eta}}, \tilde{\boldsymbol{\xi}}, \tilde{\boldsymbol{\sigma}}, \boldsymbol{v}, \boldsymbol{\mu}, \boldsymbol{\mu}_{0}\right) \\
& \dot{\tilde{\xi}}_{\rho}=\tilde{b}\left(\overline{\boldsymbol{\eta}}, \tilde{\boldsymbol{\xi}}, \overline{\boldsymbol{\sigma}}, \boldsymbol{v}, \boldsymbol{\mu}^{*}\right) \bar{\xi}_{\rho+1}+\tilde{\varphi}_{\rho}\left(\overline{\boldsymbol{\eta}}, \tilde{\boldsymbol{\xi}}, \tilde{\boldsymbol{\sigma}}, \boldsymbol{v}, \boldsymbol{\mu}, \boldsymbol{\mu}_{0}\right) \\
& \tilde{\boldsymbol{Q}}_{i}\left(\overline{\boldsymbol{\eta}}, \tilde{\xi}_{1}, \ldots, \tilde{\xi}_{i}, \tilde{\boldsymbol{\sigma}}_{1}, \ldots, \tilde{\boldsymbol{\sigma}}_{i}, \boldsymbol{v}, \boldsymbol{\mu}, \boldsymbol{\mu}_{0}\right)= \\
& \boldsymbol{Q}_{i}\left(\overline{\boldsymbol{\eta}}, \tilde{\xi}_{1}, \ldots, \tilde{\xi}_{i}+\alpha_{i-1}\left(\tilde{\xi}_{i-1}\right), \tilde{\boldsymbol{\sigma}}_{1}, \ldots, \tilde{\boldsymbol{\sigma}}_{i}, \boldsymbol{v}, \boldsymbol{\mu}, \boldsymbol{\mu}_{0}\right), \\
& \tilde{\varphi}_{i}\left(\overline{\boldsymbol{\eta}}, \tilde{\xi}_{1}, \ldots, \tilde{\xi}_{i}, \tilde{\boldsymbol{\sigma}}_{1}, \ldots, \tilde{\boldsymbol{\sigma}}_{i}, \boldsymbol{v}, \boldsymbol{\mu}, \boldsymbol{\mu}_{0}\right)= \\
& \bar{\varphi}_{i}^{\circ}\left(\overline{\boldsymbol{\eta}}, \tilde{\xi}_{1}, \ldots, \tilde{\xi}_{i}+\alpha_{i-1}\left(\tilde{\xi}_{i-1}\right), \tilde{\boldsymbol{\sigma}}_{1}, \ldots, \tilde{\boldsymbol{\sigma}}_{i}, \boldsymbol{v}, \boldsymbol{\mu}, \boldsymbol{\mu}_{0}\right)+ \\
& \alpha_{i}\left(\tilde{\xi}_{i}\right)-\frac{\partial \alpha_{i-1}\left(\tilde{\xi}_{i-1}\right)}{\partial \tilde{\xi}_{i-1}} \dot{\tilde{\xi}}_{i-1}, \\
& \tilde{\boldsymbol{Q}}_{\rho}\left(\overline{\boldsymbol{\eta}}, \tilde{\boldsymbol{\xi}}, \tilde{\boldsymbol{\sigma}}, \boldsymbol{v}, \boldsymbol{\mu}, \boldsymbol{\mu}_{0}\right)= \\
& \boldsymbol{Q}_{\rho}\left(\overline{\boldsymbol{\eta}}, \tilde{\xi}_{1}, \ldots, \tilde{\xi}_{\rho}+\alpha_{\rho-1}\left(\tilde{\xi}_{\rho-1}\right), \tilde{\boldsymbol{\sigma}}, \boldsymbol{v}, \boldsymbol{\mu}, \boldsymbol{\mu}_{0}\right), \\
& \tilde{b}\left(\overline{\boldsymbol{\eta}}, \tilde{\xi}, \tilde{\boldsymbol{\sigma}}, \boldsymbol{v}, \boldsymbol{\mu}, \boldsymbol{\mu}_{0}\right)= \\
& \bar{b}^{\circ}\left(\overline{\boldsymbol{\eta}}, \tilde{\xi}_{1}, \ldots, \tilde{\xi}_{\rho}+\alpha_{\rho-1}\left(\tilde{\xi}_{\rho-1}\right), \tilde{\boldsymbol{\sigma}}, \boldsymbol{v}, \boldsymbol{\mu}, \boldsymbol{\mu}_{0}\right), \\
& \tilde{\varphi}_{\rho}\left(\overline{\boldsymbol{\eta}}, \tilde{\xi}, \tilde{\boldsymbol{\sigma}}, \boldsymbol{v}, \boldsymbol{\mu}, \boldsymbol{\mu}_{0}\right)= \\
& \bar{\varphi}_{\rho}^{\circ}\left(\overline{\boldsymbol{\eta}}, \tilde{\xi}_{1}, \ldots, \tilde{\xi}_{\rho}+\alpha_{\rho-1}\left(\tilde{\xi}_{\rho-1}\right), \tilde{\boldsymbol{\sigma}}, \boldsymbol{v}, \boldsymbol{\mu}, \boldsymbol{\mu}_{0}\right)- \\
& \frac{\partial \alpha_{\rho-1}\left(\tilde{\xi}_{\rho-1}\right)}{\partial \tilde{\xi}_{\rho-1}} \dot{\tilde{\xi}}_{\rho-1} .
\end{aligned}
$$

are robustly input-to-state stable for all $v \in V$ and $\mu \in U$, with state $\operatorname{col}\left(\overline{\boldsymbol{\eta}}, \tilde{\boldsymbol{\sigma}}, \tilde{\xi}_{1}, \ldots, \tilde{\xi}_{\rho-1}\right)$ and input $\tilde{\xi}_{\rho}$, and have a known $C^{1}$ gain function $\kappa_{\rho}(\cdot)$.

Under Assumption 7, it can be verified that $\rho_{1}\left(\kappa_{\rho}(\cdot)\right)$ is locally Lipschitz. Then with Assumption 8, all conditions of Lemma 11.4.1 in [28] are satisfied, thus there exists

$$
\bar{\xi}_{\rho+1}=\tilde{k}\left(\tilde{\xi}_{\rho}\right)+\hat{u}
$$

such that system (28) is robustly input-to-state stable for all $\boldsymbol{v} \in V$ and $\boldsymbol{\mu} \in U$, with state $\operatorname{col}\left(\overline{\boldsymbol{\eta}}, \tilde{\boldsymbol{\sigma}}, \tilde{\xi}_{1}, \ldots, \tilde{\xi}_{\rho}\right)$ and input $\hat{u}$. As a result, the control law

$$
\bar{\xi}_{\rho+1}=\bar{k}\left(\bar{\xi}_{1}, \ldots, \bar{\xi}_{\rho}\right)=\tilde{k}\left(\tilde{\xi}_{\rho}\right)
$$

solves the global robust stabilization problem of the system (28). Hence, with transformations (25) and (27), the global robust stabilization problem of the system (21) is solvable by the control law (22).

Combining Theorem 1 and Theorem 2 gives the following result.

Theorem 3 Under Assumptions 1-8, the global robust output regulation problem of system (1) and (2) is solved by a state feedback control law (23).

\section{Illustration example}

Consider systems (1) and (2) are specified as

$$
\begin{gathered}
{\left[\begin{array}{c}
\dot{x_{1}} \\
\dot{x_{2}} \\
\dot{x_{3}}
\end{array}\right]=\left[\begin{array}{c}
x_{2}+\boldsymbol{\mu} x_{3}^{2}+\boldsymbol{\mu} x_{1} \\
-x_{1} \\
-x_{3}
\end{array}\right]+\left[\begin{array}{cc}
0 & 1 \\
-\boldsymbol{\mu}^{2} & 0 \\
0 & x_{2}
\end{array}\right] \boldsymbol{z}+} \\
{\left[\begin{array}{c}
0 \\
\boldsymbol{\mu}^{2} \\
1
\end{array}\right] u+\left[\begin{array}{cc}
0 & 0 \\
0 & -2 x_{1} \\
0 & 0
\end{array}\right] \boldsymbol{v}} \\
0=\left[\begin{array}{c}
\boldsymbol{\mu} x_{1} \\
-2 x_{1}
\end{array}\right]+\left[\begin{array}{cc}
0 & 1 \\
0 & x_{2}
\end{array}\right] \boldsymbol{z}+\left[\begin{array}{l}
0 \\
1
\end{array}\right] u+\left[\begin{array}{ll}
0 & 0 \\
2 & 0
\end{array}\right] \boldsymbol{v} \\
e(t)=x_{1}-v_{1}
\end{gathered}
$$

and

$$
\dot{\overline{\boldsymbol{\sigma}}}_{i}=\tilde{\boldsymbol{Q}}_{i}\left(\overline{\boldsymbol{\eta}}, \bar{\xi}_{1}, \ldots, \bar{\xi}_{i}, \tilde{\boldsymbol{\sigma}}_{1}, \ldots, \tilde{\boldsymbol{\sigma}}_{i}, \boldsymbol{v}, \boldsymbol{\mu}, \boldsymbol{\mu}_{0}\right)
$$

is robustly input-to-state stable for all $\boldsymbol{v} \in V$ and $\boldsymbol{\mu} \in U$, with $\overline{\boldsymbol{\sigma}}_{i}$ as state and $\operatorname{col}\left(\overline{\boldsymbol{\eta}}, \bar{\xi}_{1}, \ldots, \bar{\xi}_{i}, \overline{\boldsymbol{\sigma}}_{1}, \ldots, \overline{\boldsymbol{\sigma}}_{i-1}\right)$ as input, and has a known $C^{1}$ gain function $\kappa_{i}(\cdot)$. With Assumption 6, all conditions of Proposition 5.1 of [13] are satisfied, thus

$$
\begin{gathered}
\dot{\overline{\boldsymbol{\eta}}}=\tilde{\boldsymbol{\psi}}\left(\overline{\boldsymbol{\eta}}, \tilde{\xi}_{1}, \boldsymbol{v}, \boldsymbol{\mu}, \boldsymbol{\mu}_{0}\right) \\
\dot{\tilde{\boldsymbol{\sigma}}}_{i}=\tilde{\boldsymbol{Q}}_{i}\left(\overline{\boldsymbol{\eta}}, \tilde{\xi}_{1}, \ldots, \tilde{\xi}_{i}, \tilde{\boldsymbol{\sigma}}_{1}, \ldots, \tilde{\boldsymbol{\sigma}}_{i}, \boldsymbol{v}, \boldsymbol{\mu}, \boldsymbol{\mu}_{0}\right) \\
\dot{\tilde{\xi}}_{i}=\tilde{\xi}_{i+1}+\tilde{\varphi}_{i}\left(\overline{\boldsymbol{\eta}}, \tilde{\xi}_{1}, \ldots, \tilde{\xi}_{i}, \tilde{\boldsymbol{\sigma}}_{1}, \ldots, \tilde{\boldsymbol{\sigma}}_{i}, \boldsymbol{v}, \boldsymbol{\mu}, \boldsymbol{\mu}_{0}\right) \\
\dot{\tilde{\boldsymbol{\sigma}}}_{\rho}=\tilde{Q}_{\rho}\left(\overline{\boldsymbol{\eta}}, \tilde{\xi}_{1}, \ldots, \tilde{\xi}_{\rho}, \tilde{\boldsymbol{\sigma}}_{1}, \ldots, \tilde{\boldsymbol{\sigma}}_{\rho}, \boldsymbol{v}, \boldsymbol{\mu}, \boldsymbol{\mu}_{0}\right) \\
i=1, \ldots, \rho-1
\end{gathered}
$$

$$
\dot{\boldsymbol{v}}=\left[\begin{array}{cc}
0 & 1 \\
-1 & 0
\end{array}\right] \boldsymbol{v} .
$$

It can be verified that Assumptions 1 and 2 are satisfied with $\gamma(\boldsymbol{x})=\left[\begin{array}{ll}1 & 0\end{array}\right]$ and the system (29) has a strong relative degree $\rho=2$. By Lemma 1, we have

$$
T\left(\boldsymbol{x}, \boldsymbol{\mu}_{0}\right)=\left[\begin{array}{c}
\boldsymbol{\eta} \\
\xi_{1} \\
\xi_{2}
\end{array}\right]=\left[\begin{array}{c}
x_{3} \\
x_{1} \\
x_{2}+\boldsymbol{\mu}_{0} x_{3}^{2}
\end{array}\right]
$$

it follows that

$$
\begin{gathered}
\dot{\boldsymbol{\eta}}=-\boldsymbol{\eta}+2 \xi_{1}-2 v_{1} \\
\dot{\xi_{1}}=\xi_{2}+\left(\boldsymbol{\mu}-\boldsymbol{\mu}_{0}\right) \boldsymbol{\eta} \\
\dot{\xi_{2}}=\left(-\xi_{1}-2 \boldsymbol{\mu}_{0} \boldsymbol{\eta}^{2}\right)+\left[\begin{array}{c}
-\boldsymbol{\mu}^{2} \\
2 \boldsymbol{\mu}_{0} \boldsymbol{\eta}\left(\xi_{2}-\boldsymbol{\mu}_{0} \boldsymbol{\eta}^{2}\right)
\end{array}\right] \boldsymbol{z}+
\end{gathered}
$$




$$
\begin{gathered}
\left(\boldsymbol{\mu}^{2}+2 \boldsymbol{\mu}_{0} \boldsymbol{\eta}\right) \boldsymbol{u}+\left[\begin{array}{c}
0 \\
-2 \xi_{1}
\end{array}\right] \boldsymbol{v} \\
0=\left[\begin{array}{c}
\boldsymbol{\mu} \xi_{1} \\
-2 \xi_{1}
\end{array}\right]+\left[\begin{array}{cc}
0 & 1 \\
0 & \xi_{2}-\boldsymbol{\mu}_{0} \boldsymbol{\eta}^{2}
\end{array}\right] \boldsymbol{z}+ \\
{\left[\begin{array}{l}
0 \\
1
\end{array}\right] u+\left[\begin{array}{ll}
0 & 0 \\
2 & 0
\end{array}\right] \boldsymbol{v} .}
\end{gathered}
$$

It can be shown that Assumptions 3 and 4 are satisfied with

$$
\begin{gathered}
\alpha(\boldsymbol{v}, \boldsymbol{\mu})=0, \beta_{1}(\boldsymbol{v}, \boldsymbol{\mu})=v_{1}, \beta_{2}(\boldsymbol{v}, \boldsymbol{\mu})=v_{2}, \\
u(\boldsymbol{\mu}, \boldsymbol{v})=\boldsymbol{\mu} v_{1} v_{2}, \\
z_{1}(\boldsymbol{\mu}, \boldsymbol{v})=\boldsymbol{\mu} v_{1} v_{2}-2 v_{1} v_{2} / \boldsymbol{\mu}^{2}, z_{2}(\boldsymbol{\mu}, \boldsymbol{v})=-\boldsymbol{\mu} v_{1} .
\end{gathered}
$$

Let $\chi(\boldsymbol{v}, \boldsymbol{\mu})=\operatorname{col}\left(\beta_{2}, \beta_{3}\right)$ with $\beta_{3}(\boldsymbol{v}, \boldsymbol{\mu})=u(\boldsymbol{\mu}, \boldsymbol{v})-$ $z_{1}(\boldsymbol{\mu}, \boldsymbol{v})=2 v_{1} v_{2} / \boldsymbol{\mu}^{2}$, and

$$
\boldsymbol{\tau}_{1}(\boldsymbol{v}, \boldsymbol{\mu})=\left[\begin{array}{c}
v_{2} \\
-v_{1}
\end{array}\right], \boldsymbol{\tau}_{2}(\boldsymbol{v}, \boldsymbol{\mu})=\left[\begin{array}{c}
2 v_{1} v_{2} / \boldsymbol{\mu}^{2} \\
\left(2 v_{2}^{2}-2 v_{1}^{2}\right) / \boldsymbol{\mu}^{2}
\end{array}\right]
$$

then we have

$$
\begin{aligned}
& \boldsymbol{\Phi}_{1}=\left[\begin{array}{cc}
0 & 1 \\
-1 & 0
\end{array}\right], \quad \boldsymbol{\Psi}_{1}=\left[\begin{array}{ll}
1 & 0
\end{array}\right], \\
& \boldsymbol{\Phi}_{2}=\left[\begin{array}{cc}
0 & 1 \\
-4 & 0
\end{array}\right], \quad \boldsymbol{\Psi}_{2}=\left[\begin{array}{ll}
1 & 0
\end{array}\right] .
\end{aligned}
$$

Let

$$
\boldsymbol{M}_{1}=\boldsymbol{M}_{2}=\left[\begin{array}{cc}
0 & 1 \\
-3 & -2
\end{array}\right], \boldsymbol{N}_{1}=\boldsymbol{N}_{2}=\left[\begin{array}{c}
0 \\
0.5
\end{array}\right]
$$

it follows that

$$
\begin{gathered}
\boldsymbol{T}_{0}^{1}=\left[\begin{array}{cc}
0.125 & -0.125 \\
0.125 & 0.125
\end{array}\right] \\
\boldsymbol{T}_{0}^{2}=\left[\begin{array}{cc}
-0.0294 & -0.0588 \\
0.2353 & -0.0294
\end{array}\right] \\
\boldsymbol{\theta}_{1}(\boldsymbol{v}, \boldsymbol{\mu})=\left[\begin{array}{c}
0.5 v_{2}-0.5 v_{1} \\
-0.5 v_{2}
\end{array}\right] \\
\boldsymbol{\theta}_{2}(\boldsymbol{v}, \boldsymbol{\mu})= \\
{\left[\begin{array}{c}
\left(-0.0588 v_{1} v_{2}+0.1176 v_{1}^{2}-0.1176 v_{2}^{2}\right) / \boldsymbol{\mu}^{2} \\
\left(0.4706 v_{1} v_{2}+0.0588 v_{1}^{2}-0.0588 v_{2}^{2}\right) / \boldsymbol{\mu}^{2}
\end{array}\right] .}
\end{gathered}
$$

Thus, the internal model with output $\xi_{2}$ and $\xi_{3}=u-z_{1}$ is as follows:

$$
\begin{gathered}
\dot{\boldsymbol{\sigma}}_{1}=\boldsymbol{M}_{1} \boldsymbol{\sigma}_{1}+\boldsymbol{N}_{1} \xi_{2} \\
\dot{\boldsymbol{\sigma}}_{2}=\boldsymbol{M}_{2} \boldsymbol{\sigma}_{2}+\boldsymbol{N}_{2} \xi_{3} .
\end{gathered}
$$

The following coordinate transformations

$$
\begin{aligned}
& \overline{\boldsymbol{\sigma}}_{1}=\boldsymbol{\sigma}_{1}-\boldsymbol{\theta}_{1}(\boldsymbol{v}, \boldsymbol{\mu}) \\
& \overline{\boldsymbol{\sigma}}_{2}=\boldsymbol{\sigma}_{2}-\boldsymbol{\theta}_{2}(\boldsymbol{v}, \boldsymbol{\mu})
\end{aligned}
$$

$$
\begin{gathered}
\overline{\boldsymbol{\eta}}=\boldsymbol{\eta}-\boldsymbol{\alpha}(\boldsymbol{v}, \boldsymbol{\mu}) \\
\overline{\xi_{1}}=\xi_{1}-\beta_{1}(\boldsymbol{v}, \boldsymbol{\mu}) \\
\bar{\xi}_{2}=\xi_{2}-\boldsymbol{\Psi}_{1}\left(\boldsymbol{T}_{0}^{1}\right)^{-1} \boldsymbol{\sigma}_{1} \\
\bar{\xi}_{3}=\xi_{3}-\boldsymbol{\Psi}_{2}\left(\boldsymbol{T}_{0}^{2}\right)^{-1} \boldsymbol{\sigma}_{2}
\end{gathered}
$$

yield

$$
\dot{\overline{\boldsymbol{\eta}}}=-\overline{\boldsymbol{\eta}}+2 \bar{\xi}_{1}
$$

$$
\begin{gathered}
\dot{\overline{\boldsymbol{\sigma}}}_{1}=\left(\boldsymbol{M}_{1}+\boldsymbol{N}_{1} \boldsymbol{\Psi}_{1}\left(\boldsymbol{T}_{0}^{1}\right)^{-1}\right) \overline{\boldsymbol{\sigma}}_{1}+\boldsymbol{N}_{1} \bar{\xi}_{2} \\
\dot{\boldsymbol{\sigma}}_{2}=\left(\boldsymbol{M}_{2}+\boldsymbol{N}_{2} \boldsymbol{\Psi}_{2}\left(\boldsymbol{T}_{0}^{2}\right)^{-1}\right) \overline{\boldsymbol{\sigma}}_{2}+\boldsymbol{N}_{2} \bar{\xi}_{3} \\
\dot{\bar{\xi}}_{1}=\bar{\xi}_{2}+\boldsymbol{\Psi}_{1}\left(\boldsymbol{T}_{0}^{1}\right)^{-1}\left(\overline{\boldsymbol{\sigma}}_{1}+\boldsymbol{\theta}_{1}\right)+\left(\boldsymbol{\mu}-\boldsymbol{\mu}_{0}\right) \overline{\boldsymbol{\eta}}^{2}-\beta_{2} \\
\dot{\bar{\xi}}_{2}=\left(-\xi_{1}-2 \boldsymbol{\mu}_{0} \boldsymbol{\eta}^{2}\right)+\left[\begin{array}{c}
-\boldsymbol{\mu}^{2} \\
2 \boldsymbol{\mu}_{0} \boldsymbol{\eta}\left(\xi_{2}-\boldsymbol{\mu}_{0} \boldsymbol{\eta}^{2}\right)
\end{array}\right] \boldsymbol{z}+ \\
\left(\boldsymbol{\mu}^{2}+2 \boldsymbol{\mu}_{0} \boldsymbol{\eta}\right)\left(\bar{\xi}_{3}+\boldsymbol{\Psi}_{2}\left(\boldsymbol{T}_{0}^{2}\right)^{-1} \boldsymbol{\sigma}_{2}\right)+ \\
{\left[\begin{array}{c}
0 \\
-2 \xi_{1}
\end{array}\right] v-\boldsymbol{\Psi}_{1}\left(\boldsymbol{T}_{0}^{1}\right)^{-1} \dot{\boldsymbol{\sigma}}_{1}} \\
0=\left[\begin{array}{c}
\boldsymbol{\mu} \xi_{1} \\
-2 \xi_{1}
\end{array}\right]+\left[\begin{array}{ll}
0 & 1 \\
0 & \xi_{2}-\boldsymbol{\mu}_{0} \boldsymbol{\eta}^{2}
\end{array}\right] \boldsymbol{z}+ \\
{\left[\begin{array}{c}
0 \\
1
\end{array}\right]\left(\bar{\xi}_{3}+\boldsymbol{\Psi}_{2}\left(\boldsymbol{T}_{0}^{2}\right)^{-1} \boldsymbol{\sigma}_{2}\right)+\left[\begin{array}{ll}
0 & 0 \\
2 & 0
\end{array}\right] \boldsymbol{v} .}
\end{gathered}
$$

Under Assumption 1, we have

$$
\begin{gathered}
\dot{\overline{\boldsymbol{\eta}}}=-\overline{\boldsymbol{\eta}}+2 \bar{\xi}_{1} \\
\dot{\overline{\boldsymbol{\sigma}}}_{1}=\left(\boldsymbol{M}_{1}+\boldsymbol{N}_{1} \boldsymbol{\Psi}_{1}\left(\boldsymbol{T}_{0}^{1}\right)^{-1}\right) \overline{\boldsymbol{\sigma}}_{1}+\boldsymbol{N}_{1} \bar{\xi}_{2} \\
\dot{\overline{\boldsymbol{\sigma}}}_{2}=\left(\boldsymbol{M}_{2}+\boldsymbol{N}_{2} \boldsymbol{\Psi}_{2}\left(\boldsymbol{T}_{0}^{2}\right)^{-1}\right) \overline{\boldsymbol{\sigma}}_{2}+\boldsymbol{N}_{2} \bar{\xi}_{3} \\
\dot{\bar{\xi}}_{1}=\bar{\xi}_{2}+\boldsymbol{\Psi}_{1}\left(\boldsymbol{T}_{0}^{1}\right)^{-1}\left(\overline{\boldsymbol{\sigma}}_{1}+\boldsymbol{\theta}_{1}\right)+\left(\boldsymbol{\mu}-\boldsymbol{\mu}_{0}\right) \overline{\boldsymbol{\eta}}^{2}-\beta_{2} \\
\dot{\bar{\xi}}_{2}=\boldsymbol{\mu}^{2} \bar{\xi}_{3}+\left(4 \boldsymbol{\mu}_{0} \bar{\eta}-2 v_{2}-1\right)\left(\bar{\xi}_{1}+\beta_{1}\right)-2 \boldsymbol{\mu}_{0} \overline{\boldsymbol{\eta}}^{2}- \\
4 \boldsymbol{\mu}_{0} v_{1} \overline{\boldsymbol{\eta}}-\boldsymbol{\Psi}_{1}\left(\boldsymbol{T}_{0}^{1}\right)^{-1} \dot{\boldsymbol{\sigma}}_{1}+\boldsymbol{\mu}^{2} \boldsymbol{\Psi}_{2}\left(\boldsymbol{T}_{0}^{2}\right)^{-1} \boldsymbol{\sigma}_{2} .
\end{gathered}
$$

It can be verified that Assumptions 5-8 are satisfied. By Theorem 3, the global robust output regulation problem of (29) and (30) is solvable by

$$
\begin{gathered}
\boldsymbol{T}\left(\boldsymbol{x}, \boldsymbol{\mu}_{0}\right)=\operatorname{col}(\boldsymbol{\eta}, \boldsymbol{\xi}) \\
\dot{\boldsymbol{\sigma}}_{i}=\boldsymbol{M}_{i} \boldsymbol{\sigma}_{i}+\boldsymbol{N}_{i} \xi_{i+1}, \quad i=1,2 \\
\tilde{\xi}_{2}=\left(\xi_{2}-\boldsymbol{\Psi}_{1}\left(\boldsymbol{T}_{0}^{1}\right)^{-1} \boldsymbol{\sigma}_{2}\right)+4 e \\
\xi_{3}=-\tilde{\xi}_{2}-\left(\tilde{\xi}_{2}^{2}+2\right)^{2} \tilde{\xi}_{2}+\boldsymbol{\Psi}_{2}\left(\boldsymbol{T}_{0}^{2}\right)^{-1} \boldsymbol{\sigma}_{2} \\
u=\xi_{3}+z_{1} .
\end{gathered}
$$

The simulation conditions are selected as $\boldsymbol{x}(0)=[1 ; 2 ; 3]^{\prime}$, $\boldsymbol{v}(0)=[4 ; 5]^{\prime}, \boldsymbol{\sigma}_{1}(0)=[1 ; 2]^{\prime}, \boldsymbol{\sigma}_{2}(0)=[2 ; 1]^{\prime}, \boldsymbol{\mu} \in[13]$, $\boldsymbol{\mu}_{0}=2$. Fig. 1 shows the output error and input signal with $\boldsymbol{\mu}=1,2,3$, respectively. It can be seen that the output error $e(t)$ decays to zero and the output regulation problem of (29) and (30) is solved robustly. 

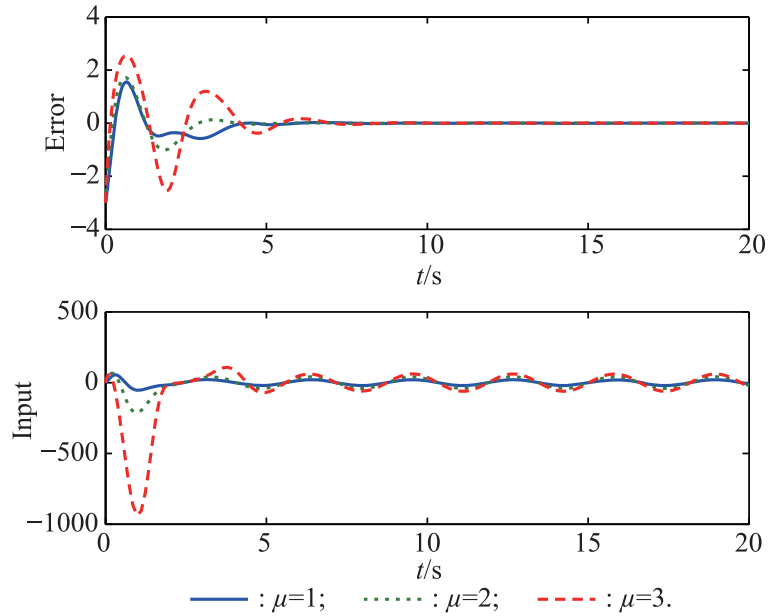

Fig. 1 Regulative error and input signal

\section{Conclusions}

This paper addresses the problem of global robust output regulation for a class of singular nonlinear systems. With the strong relative degree assumption, a global diffeomorphism map is designed to transfer the singular nonlinear system into a special singular nonlinear system. The new singular system and an internal model constitute an augmented system whose global robust stabilization solution leads to the solution of global robust output regulation problem of the original singular nonlinear system. With some appropriate assumptions, the global robust stabilization problem is solvable by a state feedback control law based on the small gain method. Therefore, the solvability conditions of the global robust output regulation problem of the singular nonlinear system is established.

\section{References}

[1] D. J. Hill, I. M. Y. Mareels. Stability theory for differential/algebraic systems with application to power systems. IEEE Trans. on Circuits and Systems, 1990, 37(11): 1416-1423.

[2] H. Krishnan, N. H. McClamroch. Tracking in nonlinear differential-algebraic control systems with applications to constrained robot systems. Automatica, 1994, 30(12): $1885-$ 1897.

[3] A. Kumar, P. Daoutidis. Feedback control of nonlinear differential-algebraic-equation systems. Aiche Journal, 1995, 41(3): $619-636$

[4] X. P. Liu, S. Celikovsky. Feedback control of affine nonlinear singular control systems. International Journal of Control, 1997, 68(4): $753-774$.

[5] X. P. Liu. Local disturbance decoupling of nonlinear singular systems. International Journal of Control, 1998, 70(5): 685 702.

[6] Z. Lu, X. Liu. Adaptive regularization for a class of nonlinear affine differential-algebraic equation systems. Proc. of the American Control Conference. 2008: 517-522.

[7] X. P. Liu,. Asymptotic output tracking of nonlinear differential-algebraic control systems. Automatica, 1998,
34(3): $393-397$

[8] J. Huang, C. F. Lin. On a robust nonlinear servomechanism problem. IEEE Trans. on Automatic Control, 1994, 39(7): $1510-1513$.

[9] H. K. Khalil. Robust servomechanism output feedback controllers for feedback linearizable systems. Automatica, 1994, 30(10): $1587-1599$.

[10] J. Huang. Asymptotic tracking and disturbance rejection in uncertain nonlinear systems. IEEE Trans. on Automatic Control, 1995, 40(6): $1118-1122$.

[11] A. Serrani, A. Isidori. Global robust output regulation for a class of nonlinear systems. Systems \& Control Letters, 2000, 39(2): $133-139$.

[12] A. Y. Memon, H. K. Khalil. Output regulation of nonlinear systems using conditional servocompensators. Automatica, 2010, 46(7): $1119-1128$.

[13] J. Huang, Z. Chen. A general framework for tackling the output regulation problem. IEEE Trans. on Automatic Control, 2004, 49(12): $2203-2218$.

[14] A. Serrani, A. Isidori, L. Marconi. Semi-global nonlinear output regulation with adaptive internal model. IEEE Trans. on Automatic Control, 2001, 46(8): 1178-1194.

[15] R. Li, H. K. Khalil. Nonlinear output regulation with adaptive conditional servocompensator. Automatica, 2012, 48(10): $2550-2559$

[16] Z. Xi, Z. Ding. Global adaptive output regulation of a class of nonlinear systems with nonlinear exosystems. Automatica, $2007,43(1): 143-149$.

[17] Z. Ding. Asymptotic rejection of unknown sinusoidal disturbances in nonlinear systems. Automatica, 2007, 43(1): 174177.

[18] Z. Zhang, A. Serrani. Adaptive robust output regulation of uncertain linear periodic systems. IEEE Trans. on Automatic Control, 2009, 54(2): 266-278.

[19] D. Xu, J. Huang. Robust adaptive control of a class of nonlinear systems and its applications. IEEE Trans. on Circuits and Systems-I: Regular Paper, 2010, 53(3): 691-702.

[20] J. Huang, J. F. Zhang. Impluse-free output regulation of singular nonlinear systems. International Journal of Control, 1998, 71(5): $789-806$.

[21] Z. Chen, J. Huang. Robust output regulation of singular nonlinear systems. Communications in Informatics and Systems, 2001, 1(4): $381-394$.

[22] Z. Chen, J. Huang. Solution of output regulation of singular nonlinear systems by normal output feedback. IEEE Trans. on Automatic Control, 2002, 47(5): 808-813.

[23] W. Wang, J. Huang. Output regulation of a class of singular nonlinear systems with the normal output feedback controller. Asian Journal of Control, 2003, 5(1): 153-159.

[24] S. Pang, J. Huang, Y. Bai. Robust output regulation of singular nonlinear systems via a nonlinear internal model. IEEE Trans. on Automatic Control, 2005, 50(2): 222-228.

[25] B. Huang, W. Lan, D. Lin, et al. Global output regulation for a class of singular nonlinear systems. Proc. of the Chinese Control Conference, 2015: 698-703.

[26] H. K. Khalil. Nonlinear systems. 3rd ed. New Jewsey: Prentice Hall, 2002.

[27] A. Isidori. Nonlinear control systems. New York: SpringerVerlag, 1995.

[28] A. Isidori. Nonlinear control systems II. New York: SpringerVerlag, 1999. 


\section{Biographies}

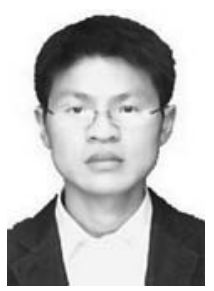

Bomin Huang was born in 1984. He received his B.S. degree in geographical sciences and M.S. degree in optics from Fujian Normal University in 2006 and 2009, respectively. He is working toward his Ph.D. degree in control theory and control engineering from Xiamen University. His research interests include nonlinear control, multi-agent systems and output regulation.

E-mail: huangbomin01@ hotmail.com

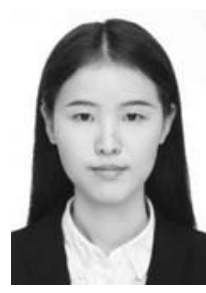

Lingmei Chen was born in 1991. She received her B.S. degree in automation from Xiamen University in 2014. Currently, she is working toward her M.S. degree in control engineering from Xiamen University. Her research interests include robust control, composite nonlinear feedback control and output regulation.

E-mail: chenlm4153@gmail.com

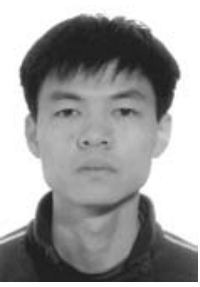

Weiyao Lan was born in 1973. He received his B.S. degree in precision instrument from Chongqing University, China, in 1995, M.S. degree in control theory and control engineering from Xiamen University, China, in 1998, and Ph.D degree in automation and computer aided engineering in 2004 from the Chinese University of Hong Kong, Hong Kong, China. From 1998 to 2000, he was a tutor at the Department of Automation, Xiamen University, Fujian, China. During 2004 to 2006, he had been a research fellow at the Department of Electrical and Computer Engineering, National University of Singapore, Singapore. Since 2007, he has been with the Department of Automation, Xiamen University, where he is currently a professor. He is a member of the 10th Technical Committee on Control Theory of Chinese Association of Automation, and is serving as an associate editor for the Transactions of the Institute of Measurement and Control. His research interests include nonlinear control theory and applications, intelligent control technology, robust and optimal control.

E-mail: wylan@xmu.edu.cn 